\title{
Nonperturbative finite-temperature Yang-Mills theory
}

\author{
Anton K. Cyrol, ${ }^{1}$ Mario Mitter, ${ }^{2}$ Jan M. Pawlowski, ${ }^{1,3}$ and Nils Strodthoff ${ }^{4}$ \\ ${ }^{1}$ Institut für Theoretische Physik, Universität Heidelberg, Philosophenweg 16, 69120 Heidelberg, Germany \\ ${ }^{2}$ Institute of Physics, NAWI Graz, University of Graz, Mozartgasse 14, 8010 Graz, Austria \\ ${ }^{3}$ ExtreMe Matter Institute EMMI, GSI, Planckstraße 1, 64291 Darmstadt, Germany \\ ${ }^{4}$ Nuclear Science Division, Lawrence Berkeley National Laboratory, Berkeley, California 94720, USA
}

(Received 22 September 2017; published 16 March 2018)

\begin{abstract}
We present nonperturbative correlation functions in Landau-gauge Yang-Mills theory at finite temperature. The results are obtained from the functional renormalisation group within a self-consistent approximation scheme. In particular, we compute the magnetic and electric components of the gluon propagator, and the three- and four-gluon vertices. We also show the ghost propagator and the ghost-gluon vertex at finite temperature. Our results for the propagators are confronted with lattice simulations and our Debye mass is compared to hard thermal loop perturbation theory.
\end{abstract}

DOI: 10.1103/PhysRevD.97.054015

\section{INTRODUCTION}

Understanding the phase structure of quantum chromodynamics still poses a major challenge. On the theoretical side, its strongly-correlated nature hampers the progress of first-principles approaches in particular at high densities. While lattice simulations struggle with the sign problem, functional methods have to address the resonant interaction structure, which requires particularly advanced truncations of the corresponding generating functionals. The tremendous progress in functional approaches to QCD has recently led to a shift from qualitative bottom-up towards quantitative top-down approaches [1-6]; see also [7-22] for reviews. In particular, the functional renormalisation group (FRG) is a first-principles method that allows for quantitative computations of the generating functional of QCD. Recently, the functional QCD (fQCD) collaboration [23] has established a comprehensive framework encompassing both, top-down [1-3] and bottom-up [24-30] approaches within the FRG framework.

In this work, we focus on thermal correlation functions of the pure gauge sector of QCD. Quantitative control over Yang-Mills theory at zero as well as finite temperature is a pivotal prerequisite for predictive investigations of the QCD phase structure with functional methods. While vacuum Yang-Mills correlation functions have been studied intensively in the past two decades [2,31-60], results for the finite-temperature correlation functions are scarce; see

Published by the American Physical Society under the terms of the Creative Commons Attribution 4.0 International license. Further distribution of this work must maintain attribution to the author(s) and the published article's title, journal citation, and DOI. Funded by SCOAP ${ }^{3}$.
[61-71] for propagator studies. For the vertices, the situation is even less satisfactory and only exploratory studies exist $[72,73]$.

The main goal of this study is to get quantitative access to the finite-temperature 1PI $n$-point functions of YangMills theory. These correlators contain all the information about the observables. For example, the resulting propagators and vertices can be used to investigate the centersymmetry phase transition in terms of the Polyakov-loop potential; see, e.g., [74-85]. Furthermore, the Debye mass, which has been studied intensively on the lattice [86-89] and hard thermal loop perturbation theory $[90,91]$ as well as with other thermal QCD approaches [92-94], can be extracted from the gluon propagator. Additionally, the correlators allow for the extraction of spectral functions and the calculation of the shear viscosity $[95,96]$.

To calculate the 1PI $n$-point functions, we perform a systematic vertex expansion of the effective action with the aim of quantitative precision, controlled by apparent convergence. The zero-temperature baseline for this calculation is provided by [2], a recent FRG study, which incorporates all tensor structures present at the classical level in a self-consistent truncation scheme. Here, we generalize this truncation to finite temperature, which includes the splitting of the correlation functions into electric and magnetic components. In particular, we provide results for the electric and magnetic gluon propagators as well as the electric and magnetic components of the three- and four-gluon vertices. For the propagators, we compare extensively to results obtained in lattice simulations. We use the Debye screening mass to determine a lower bound for the temperature range in which hard thermal loop perturbation theory can be applied straightforwardly. Furthermore, the finite-temperature behavior of 
the ghost-induced zero crossing of the three-gluon vertex is investigated. The comprehensive truncation brings along new technical challenges whose solutions are discussed. In summary, this work provides a major step towards investigations of the QCD phase structure from first principles within the functional methods.

This paper is organized as follows. In Sec. II we discuss the finite-temperature vertex expansion, order parameters, and the Debye screening mass. Section III deals with finitetemperature flows of gauge theories. We present our results in Sec. IV and discuss them in Sec. V. Finally, we summarize our findings and give an outlook in Sec. VI. Technical details and numerical checks are provided in the appendices. In particular, we confirm regulator independence in Appendix A.

\section{YANG-MILLS THEORY AT $T>0$}

We consider Euclidean Yang-Mills theory, whose classical action in general covariant gauges is given by

$S=\frac{1}{4} \int_{x} F_{\mu \nu}^{a} F_{\mu \nu}^{a}+\frac{1}{2 \xi} \int_{x}\left(\partial_{\mu} A_{\mu}^{a}\right)^{2}-\int_{x} \bar{c}^{a} \partial_{\mu} D_{\mu}^{a b} c^{b}$.

Here, $A, c$ and $\bar{c}$ denote the gluon, ghost and antighost fields and $\int_{x}=\int d^{4} x$. The gauge fixing parameter $\xi$ is taken to zero in Landau gauge, $\xi \rightarrow 0$. The field strength tensor and adjoint covariant derivative are given by

$$
\begin{aligned}
F_{\mu \nu}^{a} & =\partial_{\mu} A_{\nu}^{a}-\partial_{\nu} A_{\mu}^{a}+g f^{a b c} A_{\mu}^{b} A_{\nu}^{c}, \\
D_{\mu}^{a b} & =\delta^{a b} \partial_{\mu}-g f^{a b c} A_{\mu}^{c},
\end{aligned}
$$

where $f^{a b c}$ are the structure constants of the Lie algebra. Our notation largely follows earlier works within the fQCD collaboration [23], and we refer to [1-3,26] for further details.

\section{A. Finite-temperature vertex expansion}

Functional approaches require an approximation of the corresponding generating functional. We use a vertex expansion about the vanishing expectation values of the gluon and ghosts fields, $A_{\mu}=0$ and $c=\bar{c}=0$. These field values are solutions of the equations of motion and constitute the vacuum at vanishing temperature. The intricacies at finite temperature are discussed in more detail in the next Sec. II B. In the vertex expansion, the effective action is written as a sum over powers of the fields,

$\Gamma[\Phi]=\sum_{n} \frac{1}{n !} \bigcup_{\left\{p_{i}\right\}} \Gamma^{(n)}\left(p_{1}, \ldots, p_{n}\right) \Phi\left(p_{1}\right) \cdots \Phi\left(p_{n}\right)$,

where $\Phi=\left(A_{\mu}, \bar{c}, c\right)$ is a superfield and momentum conservation implies $\sum_{i} p_{i}=0$. The expansion coefficients in (3) are the 1PI $n$-point functions that are in field components given by

$$
\Gamma_{\Phi_{i_{1}} \cdots \Phi_{i_{n}}}^{(n)}[\Phi]=\frac{\delta^{n} \Gamma_{k}[\Phi]}{\delta \Phi_{i_{n}} \cdots \delta \Phi_{i_{1}}} .
$$

The correlation functions are expanded in terms of basis tensors $\mathcal{T}^{(i)}$ and dressing functions $\lambda^{(i)}$,

$$
\Gamma_{\Phi_{i_{1}} \ldots \Phi_{i_{n}}}^{(n)}=\sum_{i} \lambda_{\Phi_{i_{1}} \ldots \Phi_{i_{n}}}^{(i)} \mathcal{T}_{\Phi_{i_{1}} \ldots \Phi_{i_{n}}}^{(i)}
$$

At finite temperature, the vacuum $O(4)$-symmetry is replaced by $\mathbb{Z}_{2} \otimes O(3)$. This reduced symmetry implies a difference between the magnetic and electric components, which correspond to the directions that are transverse and longitudinal with respect to the heat bath. Starting from the longitudinal and transverse vacuum projection operators,

$$
\begin{aligned}
& \Pi_{\mu \nu}^{\|}(p)=\frac{p_{\mu} p_{\nu}}{p^{2}}, \\
& \Pi_{\mu \nu}^{\perp}(p)=\delta_{\mu \nu}-\Pi_{\mu \nu}^{\|}(p),
\end{aligned}
$$

we decompose four-vectors into

$$
p=\left(\begin{array}{c}
\omega_{n} \\
\vec{p}
\end{array}\right)=\left(\begin{array}{c}
2 \pi T n \\
\vec{p}
\end{array}\right),
$$

where $n \in \mathbb{Z}$ are the discrete Matsubara modes and $\omega_{n}=2 \pi T n$ the corresponding frequencies. This leads to the magnetic and electric projection operators at finite temperature,

$$
\begin{aligned}
& \Pi_{\mu \nu}^{\mathrm{M}}(p)=\left(1-\delta_{0 \mu}\right)\left(1-\delta_{0 \nu}\right)\left(\delta_{\mu \nu}-\frac{p_{\mu} p_{\nu}}{\vec{p}^{2}}\right), \\
& \Pi_{\mu \nu}^{\mathrm{E}}(p)=\Pi_{\mu \nu}^{\perp}(p)-\Pi_{\mu \nu}^{\mathrm{M}}(p) .
\end{aligned}
$$

A crucial consequence of the breaking of the vacuum $O(4)$-symmetry by (8) is the splitting of the tensor structures into electric and magnetic components. In particular, the propagators are given by

$\left[\Gamma_{A A}^{(2)}\right]_{\mu \nu}^{a b}(p)=\delta^{a b} p^{2}\left[Z_{A}^{\mathrm{M}}(p) \Pi_{\mu \nu}^{\mathrm{M}}(p)+Z_{A}^{\mathrm{E}}(p) \Pi_{\mu \nu}^{\mathrm{E}}(p)\right]$,

$\left[\Gamma_{\bar{c} c}^{(2)}\right]^{a b}(p)=\delta^{a b} p^{2} Z_{c}(p)$,

with dimensionless scalar dressing functions $1 / Z_{A}^{\mathrm{M}}$ and $1 / Z_{A}^{\mathrm{E}}$ for the magnetic and electric components of the gluon propagator. In the case of the vertices, we take only the classical tensor structures into account. Similarly to the gluon propagator, we split their dressings into electric and magnetic components. See Fig. 1 for an illustration of the constituents of our truncation and Appendixes B and C for further details. As a consequence of the restriction to classical tensors only, the tensor bases of the gluonic vertices are not complete, and the projection of the tensor equations onto the scalar dressing functions is not unique. 


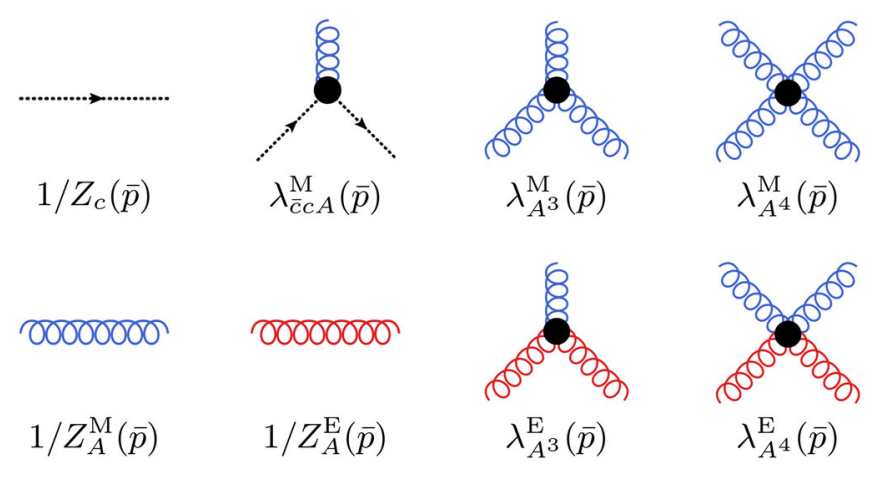

FIG. 1. Constituents of our vertex expansion. We use the classical tensors that are present in the bare action and attach magnetic (blue) and electric (red) projection operators to the gluon legs. Missing combinations, e.g., vertices with one electric leg, vanish if the Matsubara modes are set to zero and are not computed in our truncation.

We use vacuum calculations to identify uncertainties that stem from the projections in order to disentangle them from finite-temperature effects; see Appendix D for details.

Due to the breaking of $O(4)$-invariance, the scalar dressings are in general functions of the Matsubara modes and spatial momenta, e.g., $Z(p)=Z\left(\omega_{n}^{2}, \vec{p}^{2}\right)$ for a generic wave function renormalization $Z$. However, the thermal contributions to the correlation functions decay rapidly for spatial momenta and frequencies with $p^{2} \gtrsim(2 \pi T)^{2}$. Hence, the thermal correlation functions converge quickly towards their $O(4)$-symmetric vacuum counterparts for these momenta; see, e.g., Figs. 6 and 9. Consequently, the correlation functions exhibit an approximate $O(4)$ symmetry for all higher Matsubara modes, and most of the finite temperature effects are encoded in the zero mode at small spacial momenta $\vec{p}^{2} \lesssim(2 \pi T)^{2}$. Therefore, the spatial momentum dependence of the Matsubara zero modes can be used to obtain a very good approximation of the full frequency and momentum dependence via

$$
Z\left(\omega_{n}^{2}, \vec{p}^{2}\right) \approx Z\left(0, \omega_{n}^{2}+\vec{p}^{2}\right)
$$

or in short $Z(p)=Z\left(0, p^{2}\right)$. In this work, we compute the zero modes of the propagators and use (10) to close the equations. Within functional methods, this $O(4)$-symmetric approximation has been found to be quantitatively reliable for gluon [62,97] as well as quark propagators [67]. This is confirmed by lattice studies that show a slight deviation of the $O(4)$ invariance only for the first Matsubara mode at temperatures just below the critical temperature $[98,99]$.

This pattern carries over to the scalar dressings of higher order correlation functions $\lambda^{(n)}\left(p_{1}, \ldots, p_{n}\right)$. Analogously to the propagator dressings, we base our computation on the zero modes

$\lambda^{(n)}\left(\vec{p}_{1}, \ldots, \vec{p}_{i}\right)=\lambda^{(n)}\left(\omega_{n_{1}}=0, \vec{p}_{1}, \ldots, \omega_{n_{n}}=0, \vec{p}_{n}\right)$.
In contradistinction to the propagator dressings, the zero modes of the vertex dressings $\lambda^{(n)}$ depend on all $\vec{p}_{i} \cdot \vec{p}_{j}$ and not only $\vec{p}^{2}$. However, the spatial momentum dependence of the vertices is well described by a one-dimensional symmetric-point approximation in four [2] as well as three dimensions [100,101]; see also Appendix A. This leads to

$$
\lambda^{(n)}\left(\vec{p}_{1}, \ldots, \vec{p}_{n}\right) \approx \lambda^{(n)}(\bar{p}), \quad \bar{p}^{2} \equiv \frac{1}{n} \sum_{i=1}^{n} \vec{p}_{i}^{2},
$$

which allows to compute the flows of the zero modes of the vertex dressings in a quantitatively reliable approximation, cf. Fig. 12. However, the flows of the zero modes depend on the full frequency and spatial momentum dependence. Analogously to the propagator dressings, we approximate the full momentum dependence with an $O(4)$-symmetric generalization of (12),

$$
\lambda^{(n)}\left(\omega_{n_{1}}, \vec{p}_{1}, \ldots, \omega_{n_{n}}, \vec{p}_{n}\right) \approx \lambda^{(n)}(\bar{p}),
$$

where the symmetric momentum $\bar{p}$ is then given by

$$
\bar{p}^{2} \equiv \frac{1}{n} \sum_{i=1}^{n}\left(\omega_{n_{i}}^{2}+\vec{p}_{i}^{2}\right) .
$$

In summary, we use two quantitatively reliable approximations for the dressing functions: the approximate $O(4)$ invariance of all nonvanishing Matsubara modes, which allows to use only information from the lowest Matsubara mode, and the well-tested symmetric point approximation. This truncation generalizes the vacuum truncation used in [2]; see Appendix E for an explicit numerical check.

\section{B. Nontrivial vacuum and backgrounds}

As discussed in the last Sec. II A, we use a vertex expansion about vanishing field expectation values $A_{\mu}=0$ and $c=\bar{c}=0$. This necessitates a thorough discussion of the implications of this choice, in particular for comparisons to lattice results. We argue that such an expansion about vanishing background fields, i.e., Landau gauge, leads to correlation functions that agree with the lattice correlators for temperatures outside a small region around the phase transition. Furthermore, even near the phase transition, sizeable effects are only expected for correlation functions that have electric gluon legs, the electric gluon propagator being their most prominent representative. This becomes most evident by investigating the relation of the physical solution of the equation of motion in nonvanishing gluon background fields and the Polyakov loop, the canonical order parameter of the confinementdeconfinement phase transition. For the convenience of the reader, the first two parts briefly review corresponding relevant advances in nonperturbative functional approaches [74-79,102]; see [65,80-85] for further applications. 


\section{Correlation functions}

To facilitate the discussion, we use the background extension of Landau gauge,

$$
\left(\partial_{\mu}-\mathrm{i} g \bar{A}_{\mu}\right) a_{\mu}=0 \quad \text { with } \quad A_{\mu}=\bar{A}_{\mu}+a_{\mu},
$$

called Landau-deWitt gauge. Here, $\bar{A}_{\mu}$ is a general background and $a_{\mu}$ is the quantum fluctuation field. In this formulation, the effective action is gauge invariant under background gauge transformations, which allows for a simpler interpretation of physical backgrounds as well as simpler technical implementations. Besides being a functional of $\Phi=\left(a_{\mu}, \bar{c}, c\right)$, the effective action depends now also on the background $\bar{A}$. Accordingly, the vertices $\Gamma^{(n)}[\bar{A}, \Phi]$ are correlation functions in the background

$$
\Phi=\langle\hat{\Phi}\rangle_{J(\bar{A}, \Phi)} \cdot
$$

Here, we have introduced the background current, which satisfies $J(\bar{A}, \Phi)=\delta \Gamma / \delta \Phi$. The correlation functions in the absence of external sources, $J(\bar{A}, \Phi)=0$, are then given by $\Gamma^{(n)}\left[\bar{A}, \Phi_{\mathrm{EoM}}\right]$, where $\Phi_{\mathrm{EoM}}$ is a solution of the equation of motion in the chosen background $\bar{A}$,

$$
\left.\frac{\delta \Gamma[\bar{A} ; \Phi]}{\delta \Phi}\right|_{\Phi_{\mathrm{EoM}}}=0
$$

In general, this conditions yields stationary points of the effective action. In particular, the expansion point $(\bar{A}, \Phi)=0$ satisfies (17), but does not necessarily single out the physical minimum. In contrast, the physical correlators that correspond to scattering amplitudes are obtained at the physical solution of the equation of motion (17), i.e., the minimum of the effective action $\left(\bar{A}, \Phi_{\min }[\bar{A}]\right)$. This is also the field value about which the vertex expansion is expected to be most stable and converge most rapidly. Furthermore, only an expansion around the physical solution of the equation of motion allows for a direct comparison with correlation functions from lattice simulations, since the latter are measured on the physical ground state. In general, any other expansion point requires information about higher correlation functions in order to evaluate $\Gamma^{(n)}\left[\bar{A}, \Phi_{\min }\right]$. In particular, in a vertex expansion with expansion point $(\bar{A}, \Phi)=0$, the inverse propagator is given by

$$
\begin{aligned}
\Gamma^{(2)}\left[\bar{A}, \Phi_{\min }\right]= & \sum_{n} \frac{1}{n !} \bigcup_{\left\{p_{i}\right\}} \Gamma^{(2+n)}[\bar{A}, 0]\left(p_{1}, \ldots, p_{n}\right) \\
& \times \Phi_{\min }\left(p_{1}\right) \cdots \Phi_{\min }\left(p_{n}\right),
\end{aligned}
$$

where we suppressed external momentum arguments. Therefore, we expect deviations between the correlation functions $\Gamma^{(n)}[0,0]$, computed in this work, and those from lattice simulations. However, these differences are sizeable only if the momentum scales of the solution $\left(\bar{A}, \Phi_{\min }\right) \neq 0$ are of the same order as $T_{c}$, the characteristic scale of the finite temperature Yang-Mills system. Only in this case, the higher correlation functions would lead to noticeable contributions in (18).

We can utilize the background field to achieve a technical simplification. Since it is arbitrary, we can choose $\bar{A}=\bar{A}_{\min } \equiv\langle\bar{A}\rangle$ such that

$$
\Phi_{\min }\left[\bar{A}_{\text {min }}\right]=0 .
$$

For this particular choice, the background carries all the nontrivial information about the ground state, whereas the (classical) fluctuation field vanishes on the equation of motion. The physical correlators are then given by $\Gamma^{(n)}[\langle\bar{A}\rangle, 0]$. In particular, the inverse propagator (18) for the gluon is then given by

$$
\Gamma_{A A}^{(2)}\left[\bar{A}, \Phi_{\min }\right]=\Gamma_{A A}^{(2)}[\langle\bar{A}\rangle, 0] .
$$

Semi-perturbative studies in the Curci-Ferrari model for Yang-Mills theory confirm that the background has large effects on the electric propagator at temperatures close to the phase transition [65]. Furthermore, the calculation of quantitatively correct values for the chiral phase transition temperature as well as its observed coincidence with the confinement-deconfinement crossover temperature require to take into account such a nontrivial minimum [76]. Finally, such a consistent treatment was also required for the description of the Roberge-Weiss transition [76] as well as the study of criticality in $S U(2)$ Yang-Mills theory $[75,102]$.

\section{Order parameters}

A further advantage of the background $\langle\bar{A}\rangle$ is its relation to the Polyakov loop $[74,76,78,79]$, the standard order parameter of the confinement-deconfinement phase transition. The traced Polyakov loop is expressed as a correlator of the temporal gauge field with

$$
\begin{aligned}
& L\left[A_{0}\right]=\frac{1}{N_{c}} \operatorname{tr} P\left[A_{0}\right], \\
& P\left[A_{0}\right]=\mathcal{P} e^{\mathrm{i} g \int_{0}^{\beta} d t A_{0}(t, \vec{x})} \equiv e^{2 \pi \mathrm{i} \varphi\left[A_{0}\right]} .
\end{aligned}
$$

Here, $\mathcal{P}$ stands for path ordering, and the functional $\varphi\left[A_{0}\right]$ is the gauge-covariant algebra element of the Polyakov loop. It transforms as $\varphi \rightarrow U \varphi\left[A_{0}\right] U^{\dagger}$ under time-periodic gauge transformation $U \in S U(N)$. This entails that the eigenvalues of $\varphi\left[A_{0}\right]$ are gauge invariant, and consequently the eigenvalues of its expectation value $\bar{\varphi} \equiv\left\langle\varphi\left[A_{0}\right]\right\rangle$ are observables. This expectation value, as well as $L[\langle\varphi\rangle]$, are order parameters for the confinement-deconfinement 


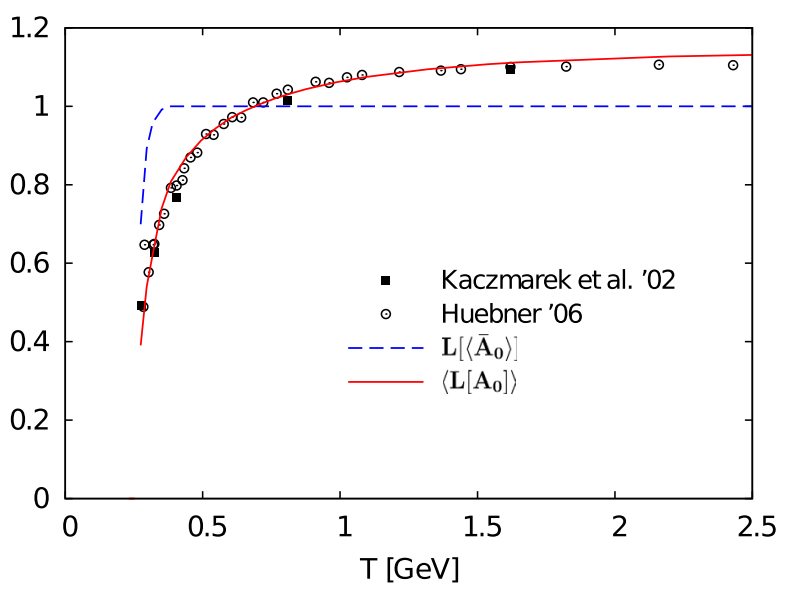

FIG. 2. Expectation value of $\left\langle L\left[A_{0}\right]\right\rangle$ versus $L\left[\left\langle\bar{A}_{0}\right\rangle\right]$ from [79]. Both observables are order parameters for the confinementdeconfinement phase transition. Moreover, $L\left[\left\langle\bar{A}_{0}\right\rangle\right]=1$ entails $\left\langle\bar{A}_{0}\right\rangle=0$.

phase transition. In Polyakov gauge, the expectation value of the algebra element takes the particularly simple form

$$
\bar{\varphi}=\beta g \bar{A}_{0},
$$

for a given background $\bar{A}_{0}$. Due to background gauge invariance, the eigenvalues of $\bar{\varphi}$ can therefore be calculated from the eigenvalues of $\bar{A}_{0}$ in any background gauge. In particular, the effective potential, $V\left[\bar{A}_{0}\right]$, of Landau-deWitt gauge carries thus the full information about the eigenvalues of the expectation value of $\varphi$.

In conclusion, the effective potential $V\left[\bar{A}_{0}\right]$ is an order parameter potential for center symmetry. The gauge invariant observables, $\left\langle L\left[A_{0}\right]\right\rangle$ and $L\left[\left\langle\bar{A}_{0}\right\rangle\right]$, or equivalently also $\left\langle\bar{A}_{0}\right\rangle$, serve as order parameters for the confinementdeconfinement phase transition; see Fig. 2. Therefore, the vanishing of $\left\langle L\left[A_{0}\right]\right\rangle$ in the confined phase relates to a nonvanishing value for $\left\langle\bar{A}_{0}\right\rangle$. This has recently been demonstrated explicitly in a self-consistent vertex expansion scheme, which has been used for the first computation of $\left\langle L\left[A_{0}\right]\right\rangle$ within functional methods [79]. Finally, the electric propagator $\left\langle A_{0}(p) A_{0}(-p)\right\rangle$ is closely related to the propagator of an order parameter field, and as such should show critical properties; see [78]. Hence, we expect the electric correlators to be affected most by the background field.

\section{Comparison to lattice simulations}

The previous discussion of the nontrivial $\bar{A}_{0}$ background and its relation to the order parameter of the confinementdeconfinement phase transition allows us to derive a theoretical estimate of the temperature range, in which our present results potentially deviate from the respective lattice results due to the different background configurations. The first important piece of information is given by the fact that the order parameter $L\left[\left\langle\bar{A}_{0}\right\rangle\right]$ approaches unity rapidly for temperatures above the phase transition temperature; see Fig. 2. This in stark contrast to the Polyakov loop $\left\langle L\left[A_{0}\right]\right\rangle$, which is usually calculated in lattice simulations. The latter reaches its asymptotic value only for $T \gg T_{c}$, which can be understood from fluctuation effects [79]. The fact that $L\left[\left\langle\bar{A}_{0}\right\rangle\right]$ quickly approaches unity above the transition temperature can be formulated as the more precise statement,

$$
\left\langle\bar{A}_{0}\right\rangle \approx 0 \quad \text { if } T \gtrsim 1.3 T_{c} .
$$

As a consequence, we can expect quantitative effects due to the nontrivial background only at temperatures $T \lesssim 1.3 T_{c}$. The most immediate effect of this nontrivial background is a shift in the Matsubara frequencies $\omega_{n} \rightarrow \omega_{n} \pm 2 \pi T \nu_{i}$, where $\nu_{i}$ are the eigenvalues of $\bar{\varphi}$, or equivalently of $\beta g\left\langle\bar{A}_{0}\right\rangle /(2 \pi)$. Rotating the constant field into the Cartan sub-algebra, these are given by

$\nu_{S U(2)}=\left\{0, \pm \varphi_{3}\right\}, \quad \nu_{S U(3)}=\left\{0,0, \pm \varphi_{3}, \pm \frac{\varphi_{3} \pm \sqrt{3} \varphi_{8}}{2}\right\}$,

in $S U(2)$ and $S U(3)$; see, e.g., [79]. However, for $T \lesssim 0.5 T_{c}$ the effect of the shifts of the Matsubara frequencies is suppressed by the zero temperature gapping $m_{\text {gap }}$ of the gluon propagator $2 \pi \nu_{i} T / m_{\text {gap }} \ll 1$. Therefore, we expect sizeable effects due to the nontrivial background only in the regime

$$
T \in\left(0.5 T_{c}, 1.3 T_{c}\right)
$$

and in particular in the electric gluon propagator.

\section{Debye screening mass}

Gluons are screened at high temperatures by the standard thermal Debye mass. However, also in the confined phase, they posses a finite screening mass. Our nonperturbative results allow to compute a screening mass also below the critical temperature. We extract it from the zero mode of the electric gluon propagator, $\left.G_{T}^{\mathrm{E}}(p)=\left\langle A_{0}(p) A_{0}(-p)\right)\right\rangle$, whose computation is detailed below in Sec. III. To this end, we Fourier transform the propagator,

$$
G_{T}^{\mathrm{E}}(x)=\int_{-\infty}^{\infty} \frac{d p}{2 \pi} G_{T}^{\mathrm{E}}(p) e^{\mathrm{i} p x} .
$$

At high temperatures, the screening mass can then be extracted from the exponential decay at large distances,

$$
\lim _{x \rightarrow \infty} G_{T}^{\mathrm{E}}(x)=c_{e} \exp \left(-m_{s} x\right) .
$$




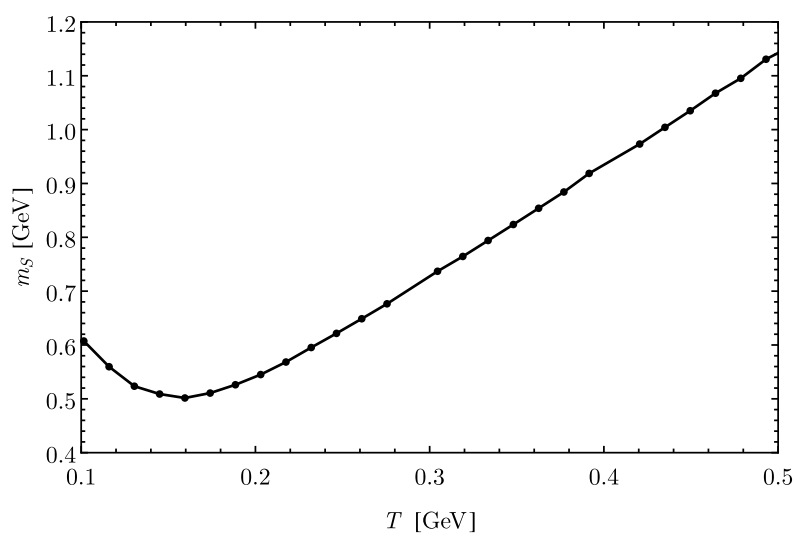

(a)

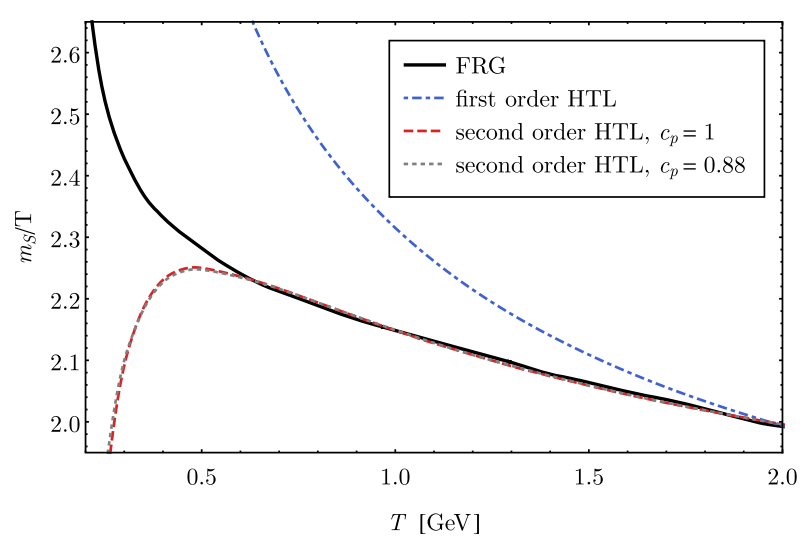

(b)

FIG. 3. Debye screening mass $m_{s}$; see Fig. 18 for the fits of (27) to $G_{T}(x)$. (a) Screening mass $m_{s}$ in units of GeV at low temperatures. (b) Dimensionless Debye screening $m_{s} / T$ mass at high temperatures in comparison with leading order perturbation theory (28) and the Arnold-Yaffe prescription (29) for accommodating beyond leading order effects [92].

The screening mass $m_{s}$ obtained with (27) is shown in Fig. 3. The large distance behavior of $G_{T}^{\mathrm{E}}(x)$ and the fits by (27) are provided in Appendix I. The left panel shows that the screening mass is finite across the phase transition and possesses a minimum at some finite temperature. Perturbatively, the Debye mass is given to leading order by

$$
m_{D}^{0}=\sqrt{\frac{N}{3}} g_{T} T+\mathcal{O}\left(g_{T}^{2} T\right) .
$$

A prescription for taking higher-order effects into account has been proposed by [92]

$m_{D}=m_{D}^{0}+\left(c_{D}+\frac{N}{4 \pi} \ln \left(\frac{m_{D}^{0}}{g_{T}^{2} T}\right)\right) g_{T}^{2} T+\mathcal{O}\left(g_{T}^{3} T\right)$

In order to compare our screening mass to the expressions (28) and (29), we have to determine $g_{T}$ and $c_{D}$. We use

$$
g_{T}=\sqrt{4 \pi \alpha_{A^{3}}^{\mathrm{E}}\left(T, p=c_{p} 2 \pi T\right)},
$$

and fit $c_{D}$ at large temperatures to our result since it is not computable within perturbation theory. The running coupling of the (electric) three-gluon vertex $\alpha_{A^{3}}^{\mathrm{E}}$ is introduced below in (37).

As shown in Fig. 3(b), the Arnold-Yaffe Debye mass agrees almost perfectly with our nonperturbative result down to $T \approx 0.6 \mathrm{GeV}$. In contrast, the leading-order Debye mass deviates instantly from our result. By default, we set $c_{p}=1$ in (30) because this is the scale that is expected to contribute most. This yields for the nonperturbative constant $c_{D}=0.100(3)$. To substantiate the choice $c_{p}=1$, we also leave it as a free fit parameter and find $c_{p}=0.88(64)$, where the nonperturbative constant $c_{D}=0.105(30)$ changes only within the fit uncertainties. The effect on the resulting Debye mass is negligible; see Fig. 3(b). The excellent agreement at very high temperatures provides a nontrivial check of the calculations. Further physical consequences are discussed in Sec. V B.

\section{METHOD}

In this section, we discuss the flow equations, the implications of the regulator term at nonvanishing RG scale, and we provide details on the numerical implementation.

\section{A. FRG flows}

The functional renormalization group in Wetterich's formulation [103] allows to integrate momentum-shell contributions to the effective action in the Wilsonian spirit. To this end, a scale- and momentum-dependent mass term is added to the classical action,

$$
\Delta S_{k}=\int_{x} \frac{1}{2} A_{\mu}^{a} R_{k, \mu \nu}^{a b} A_{\nu}^{b}+\int_{x} \bar{c}^{a} R_{k}^{a b} c^{b} .
$$

The regulator term suppresses quantum as well as thermal fluctuations at momenta below the RG scale $k$. Taking the derivative with respect to the scale $k$ leads to the Wetterich equation for the generalized effective action $\Gamma_{k}$. This flow equation interpolates between the classical $\Gamma_{k \rightarrow \Lambda \rightarrow \infty}=S$ and the 1PI effective action $\Gamma_{k \rightarrow 0}=\Gamma$. For the pure gauge theory at finite temperature, the Wetterich equation reads

$$
\partial_{t} \Gamma_{k}[\Phi]=\bigvee_{q} \frac{1}{2} G_{k, \mu \nu}^{a b}[\Phi] \partial_{t} R_{k, \nu \mu}^{b a}-\bigcup_{q} G_{k}^{a b}[\Phi] \partial_{t} R_{k}^{b a},
$$

where $t=\ln (k / \Lambda)$ denotes the RG time and 


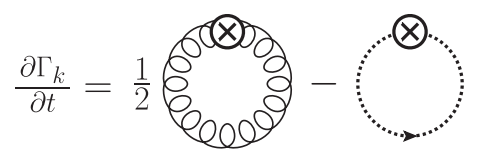

FIG. 4. Graphical representation of the Wetterich equation. Wiggly (dotted) lines represent the dressed gluon (ghost) propagators. The cross in the circle denotes the regulator insertion $\partial_{t} R_{k}$ of the corresponding field type.

$$
G_{k}[\Phi]=\frac{1}{\Gamma^{(2)}[\Phi]+R_{k}} .
$$

Using the Matsubara formalism, the momentum integral in (32) is given by

$$
y_{q}=\int \frac{d^{3} q}{(2 \pi)^{3}} T \sum_{n},
$$

where $q_{0}=2 \pi T n \equiv \omega_{n}$. A graphical representation of the Wetterich equation for the effective action is shown in Fig. 4. The truncated flow equations for the correlation functions that are obtained by taking functional derivatives of (32) are displayed in Fig. 5.

Instead of the flat regulator [104] used in the vacuum computation [2], we use an exponential regulator. As demonstrated in Appendx A, the results for the correlation functions do not depend on this choice within our error bars. However, analytic regulators such as the exponential regulator are better suited for numerical calculations of thermodynamic quantities since they carry the thermal exponential decay with the cutoff scale $\sim e^{-c k / T}$ in the flow [62,97]; see [105] for a detailed study.

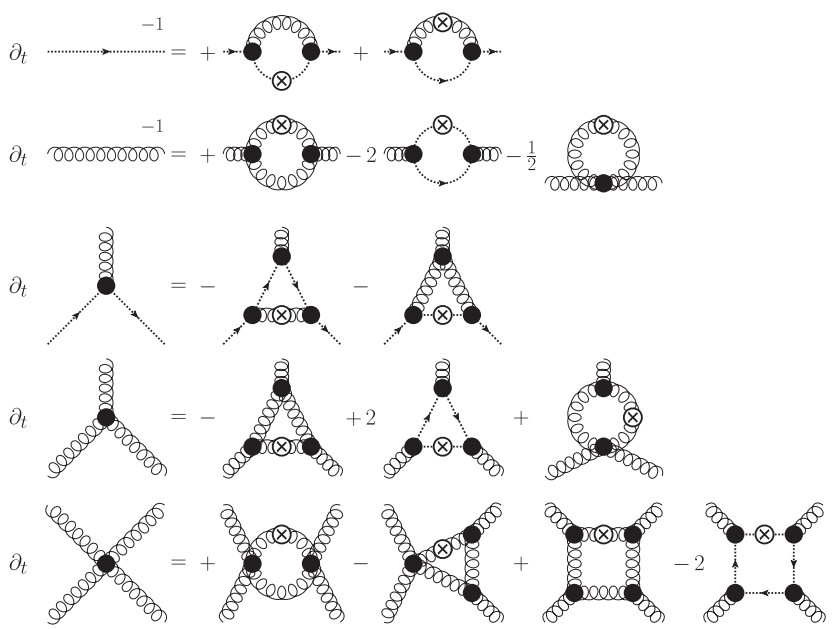

FIG. 5. Truncated equations of all computed $n$-point functions. Permutations of external legs and regulator insertions are omitted in the vertex equations. The gluon lines represent the sum over electric and the magnetic propagators, cf. (9). They are connected to the corresponding magnetic and electric components of the vertices; see also Fig. 1.
To reduce the numerical effort of the finite-temperature calculation, we exploit the degeneracy of the dressings for $k \gg 2 \pi T$. We integrate the finite-temperature flow starting from the nontrivial zero-temperature effective action at

$$
\Lambda_{T}=\max \left(\lambda 2 \pi T, \Lambda_{T}^{\min }\right),
$$

with $\lambda=4$ and $\Lambda_{T}^{\min }=1 \mathrm{GeV}$; see Appendix F for details.

\section{B. Renormalization and mSTIs}

In the presence of a regulator, the BRST-symmetry leads to modified Slavnov-Taylor identities (mSTIs) for nonvanishing $\mathrm{RG}$ scales, $k>0$ [2,8,106-111]. The additional terms are generated by the BRST-variation of the regulator term and have a one loop form. They are similar in form and structure to the flow equation itself. The latter encodes the breaking or flow of scale invariance while the former encode the breaking or flow of BRST symmetry. The resulting $\mathrm{mSTIs}$ reduce to the standard STIs in the limit of vanishing RG scale, $k \rightarrow 0$, similar to the removal of the explicit breaking of scale invariance due to the regulator. Therefore, in both cases the underlying symmetry is restored in the limit of vanishing RG scale $k \rightarrow 0$. We emphasize that any regularization scheme in momentum space leads to such a modification of BRST symmetry in terms of modified STIs. This is also well known from perturbation theory, where a cutoff regularization, amongst other modifications, requires a gluon mass counter term in order to guarantee gauge invariance. Modified STIs are also present within other functional methods such as nonperturbative DSE and $n \mathrm{PI}$ approaches that rely on numerical momentum integrations.

To take the modification of the STIs of the vertices into account, we choose constant vertex dressings $\lambda_{\bar{c} c A}, \lambda_{A^{3}}$ and $\lambda_{A^{4}}$ at the cutoff scale, $k=\Lambda$, such that the STIs for the running couplings,

$$
\alpha_{\bar{c} c A}(\mu)=\alpha_{A^{3}}(\mu)=\alpha_{A^{4}}(\mu) \equiv \alpha(\mu),
$$

are fulfilled at $\mu=20 \mathrm{GeV}, k=0$. Here, the running couplings in (36) are obtained from the classical vertices,

$$
\begin{aligned}
\alpha_{\bar{c} c A}(\bar{p}) & =\frac{\left(\lambda_{\bar{c} c A}(\bar{p})\right)^{2}}{Z_{A}(\bar{p}) Z_{c}^{2}(\bar{p})}, \\
\alpha_{A^{3}}(\bar{p}) & =\frac{\left(\lambda_{A^{3}}(\bar{p})\right)^{2}}{Z_{A}^{3}(\bar{p})}, \\
\alpha_{A^{4}}(\bar{p}) & =\frac{\lambda_{A^{4}}(\bar{p})}{Z_{A}^{2}(\bar{p})},
\end{aligned}
$$

with the symmetric momentum configuration $\bar{p}$.

The $\mathrm{mSTI}$ of the gluon propagator implies a nonvanishing longitudinal gluon mass term at the cutoff scale [106]. In the perturbative regime, it can be shown that the transverse mass agrees with the longitudinal one; for 
details, see [2]. However, while the longitudinal mass parameter vanishes at $k=0$, the transverse mass term encodes the gapping of the transverse gluon propagator at $k=0$. At the initial UV cutoff scale $k=\Lambda$ the gluon mass parameter is uniquely determined by the mSTI and cannot be chosen freely. Its precise determination is at the root of confinement, which is encoded in the transverse mass gap at vanishing cutoff scale. Since the mass parameter is proportional to the cutoff, $m_{\Lambda}^{2} \propto \alpha(\Lambda) \Lambda^{2}$, quadratic precision is required in its determination from the mSTI. The solution of this quadratic fine tuning problem requires both, a BRST-consistent quantitative level of the approximation, as well as sufficient numerical precision. Consequently, in truncated systems of flow equations, its computation from the mSTI at the required precision level is extremely challenging. The above brief discussion is detailed in [2]. Note that these statements hold also for other functional methods such as DSE and $n$ PI approaches.

In the present work we utilize that it is possible to uniquely determine the gluon mass parameter by demanding a solution of the scaling type; for details, see again [2]. We exploit that this also holds at finite temperature. Requiring scaling in the magnetic sector provides us with a unique value for the gluon mass parameter at each temperature. This procedure resolves the necessity of a BRST-consistent level of the approximation, but still requires quadratic precision in the fine-tuning. Further details are provided in Appendix G.

\section{Numerical implementation}

To solve the system of coupled flow equations, we use the tools established by the fQCD collaboration [23]. The tensorial flow equations are derived with DoFun [112]. Subsequently, the projected equations are traced with FormTracer [113], a Mathematica package that uses FORM [114-117] and has native support for finite-temperature applications. The output is exported as optimized C++ code, which is then used within the computational framework of

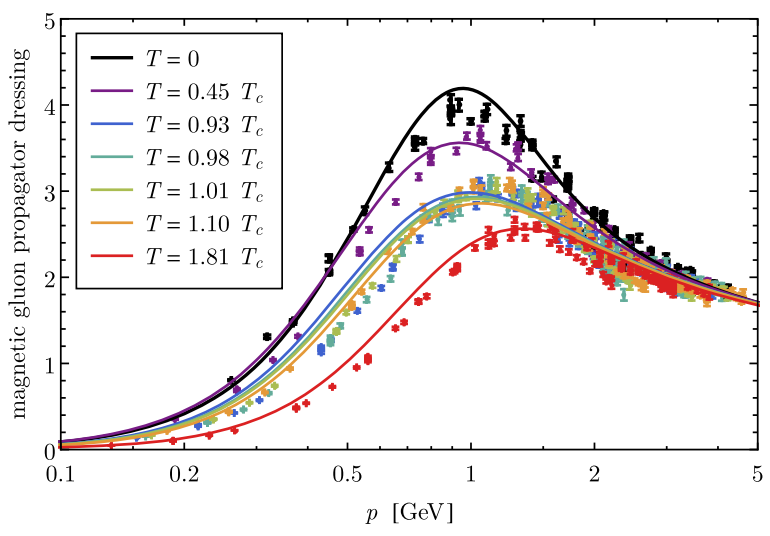

(a) Comparison with $S U(2)$ results $[69,103]$ the fQCD collaboration. The latter uses the adaptive ordinary differential equation solver from the Boost library [118] and the adaptive multidimensional integration routine from [119], which implements [120,121].

In the derivation of the equations, tracing the four-gluon vertex equation, and in particular the gluon box diagrams, is the most challenging part. To this end, we generate FORM files with FormTracer [113] for each of the twelve permutations of the box diagrams. Executing one of these with FORM can take up to eight core days and intermediate expressions reach more than $1 \mathrm{~TB}$ in size. Since the resulting expressions are still very large, we sum all permutations, factorize all dressing functions and then use the simultaneous optimization feature of FORM's optimization routine [116] in combination with a parallelized version of FORM [122] to optimize the result. Concerning the numerical computation, integrating the flow once takes roughly one day on an ordinary quad-core desktop computer. This has to be done multiple times for each temperature due to the gluon mass parameter determination.

\section{RESULTS}

The main results are displayed in Figs. 6-11. We show results for the magnetic and electric dressing functions of propagators and vertices for various temperatures. For all correlators we find that the magnetic and electric dressings coincide for momenta $p \gg 2 \pi T$, and become degenerate with the vacuum dressings. This is required by the recovery of $O(4)$ invariance. The convergence towards the vacuum dressings for small temperatures is explicitly checked in Appendix E. This apparently obvious property is actually nontrivial within frequency and momentum-dependent nonperturbative truncations.

We compare our gluon propagators to $S U(2)[69,123]$ and $S U(3)$ [71] lattice results in Figs. 6 and 7. This comparison requires the setting of a relative scale as well as renormalization, detailed in Appendix $\mathrm{H}$. As a consequence, a potential relative offset of functional and lattice

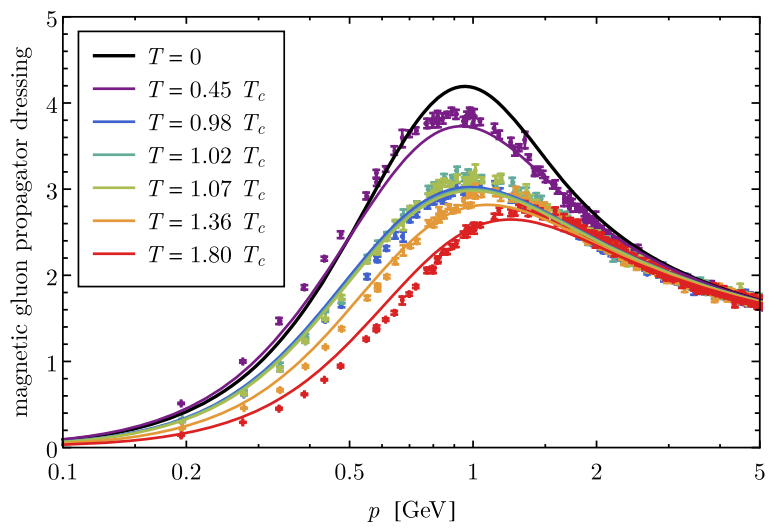

(b) Comparison with $S U(3)$ results [71].

FIG. 6. Magnetic gluon propagator dressing, (9). 


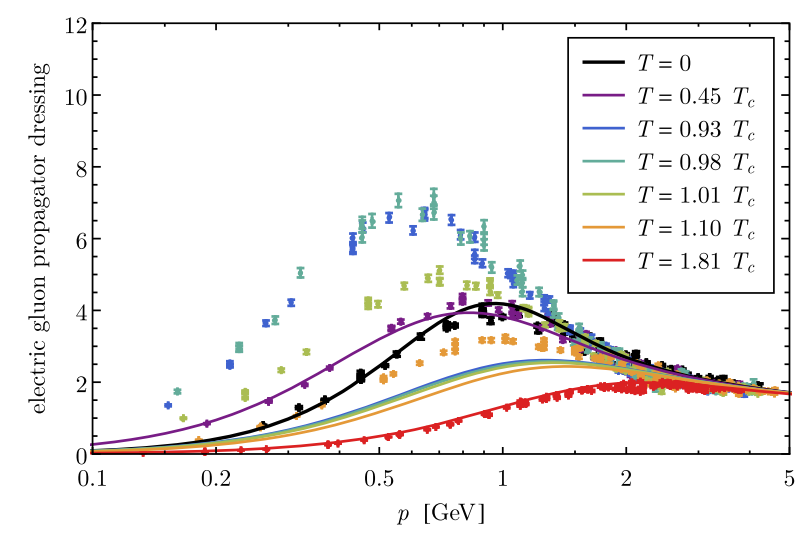

(a) Comparison with $S U(2)$ results [69, 103].

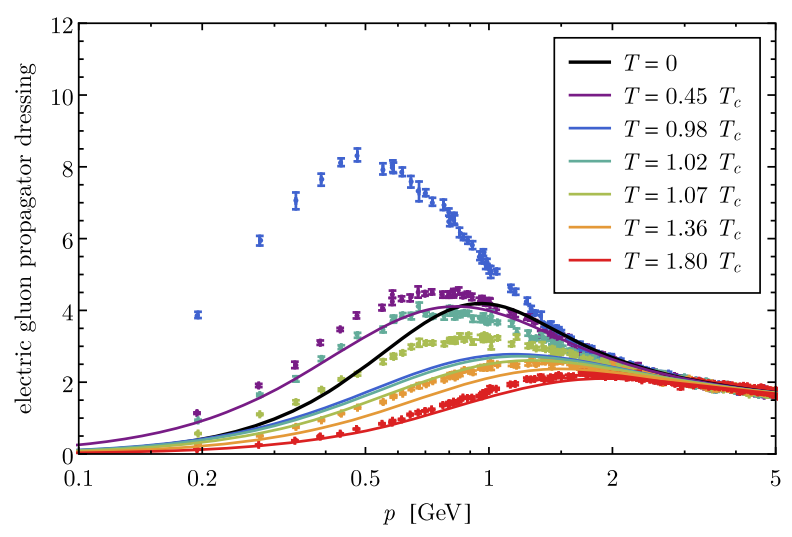

(b) Comparison with $S U(3)$ results [71].

FIG. 7. Electric gluon propagator dressing, (9).

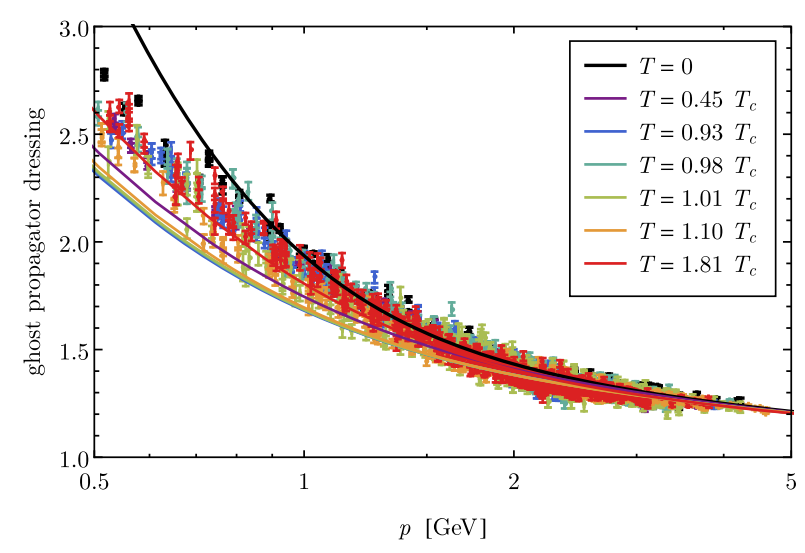

(a) Ghost propagator dressing $1 / Z_{c}(p)$.

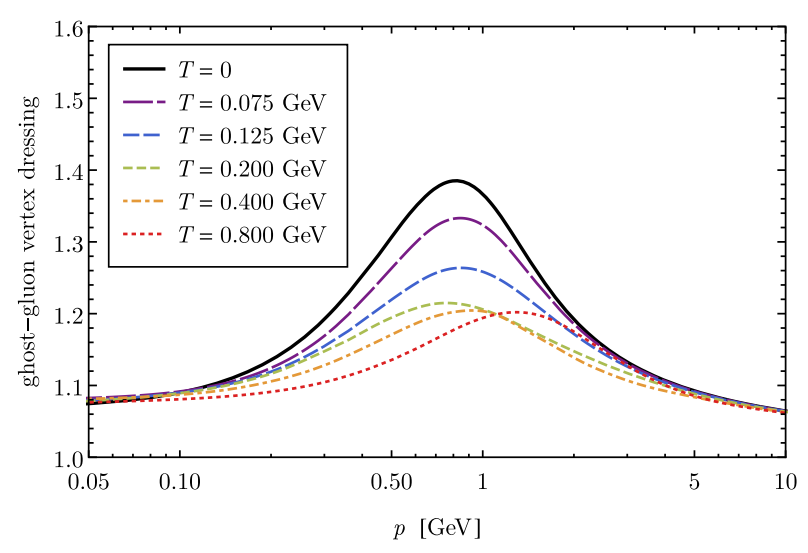

(b) Magnetic ghost-gluon vertex dressing $\lambda_{\bar{c} c A}^{\mathrm{M}}(p)$.

FIG. 8. Ghost propagator dressing, (9), compared to $S U(2)$ lattice results $[69,123]$ and ghost-gluon vertex, (B1).

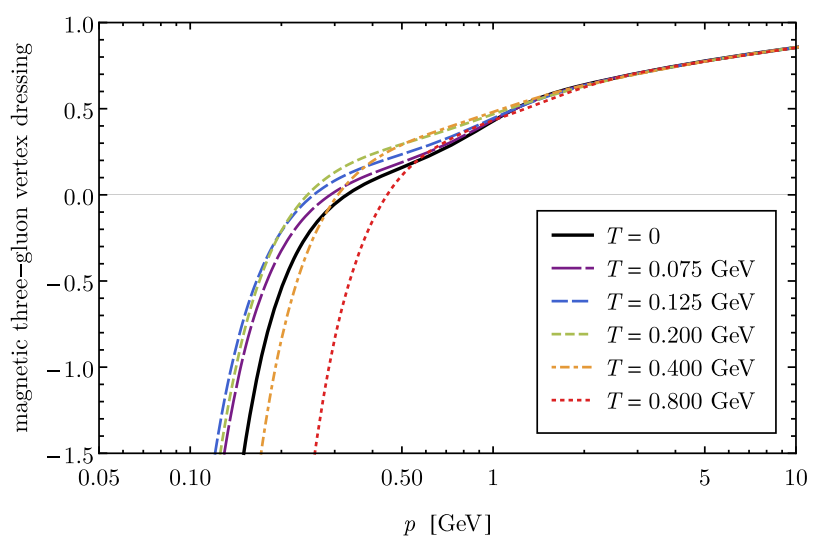

(a) Magnetic three-gluon vertex dressing $\lambda_{A^{3}}^{\mathrm{M}}(p)$.

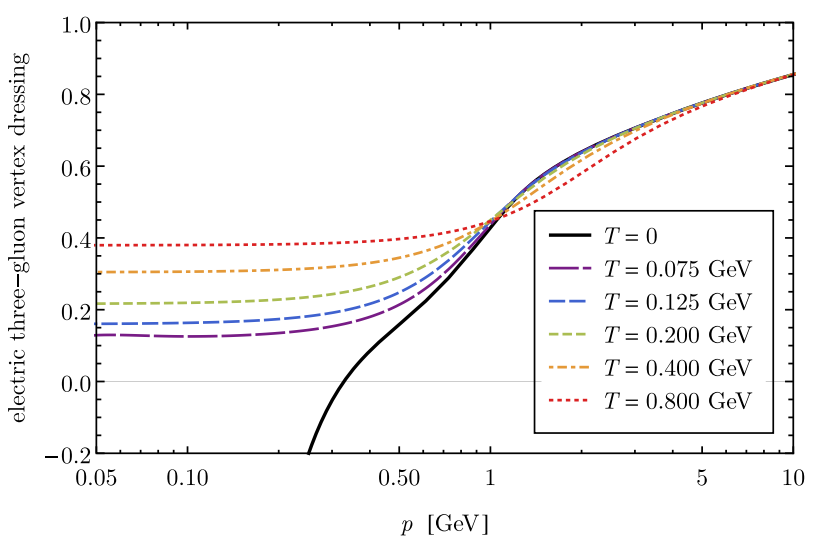

(b) Electric three-gluon vertex dressing $\lambda_{A^{3}}^{\mathrm{E}}(p)$.

FIG. 9. Temperature dependence of the three-gluon vertex dressing, (B2).

results has to be considered in addition to the systematic errors of the truncation, when juxtaposing the results from the different calculations. The comparison with $S U(2)$ as well as $S U(3)$ lattice data is legitimate because the truncation used in this work yields only a trivial dependence on the gauge group. This is the case because the color traces can be taken without specifying the gauge group $[113,126]$, and the only group constant appearing in the 


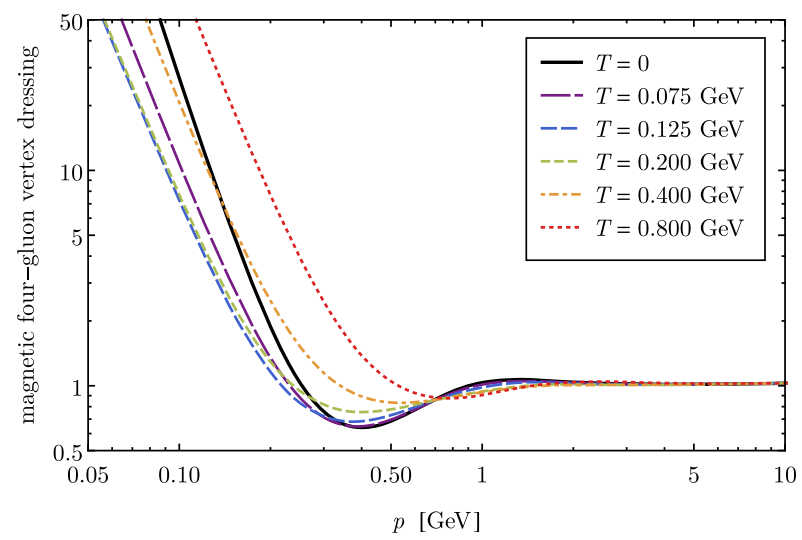

(a) Magnetic four-gluon vertex dressing $\lambda_{A^{4}}^{\mathrm{M}}(p)$.

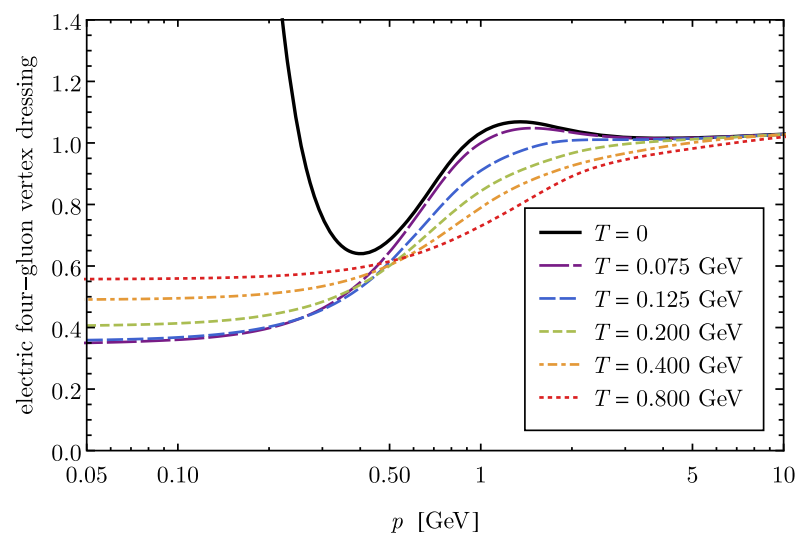

(b) Electric four-gluon vertex dressing $\lambda_{A^{4}}^{\mathrm{E}}(p)$.

FIG. 10. Temperature dependence of the four-gluon vertex dressing, (B3).

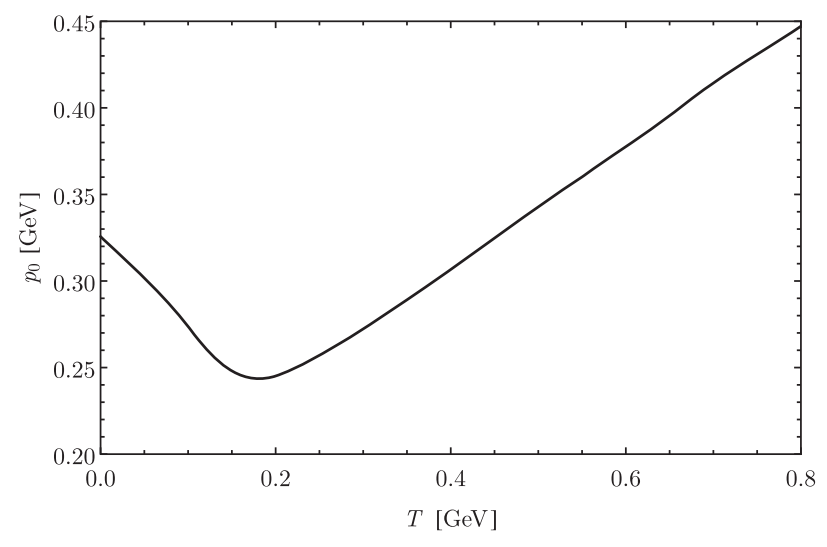

FIG. 11. Temperature dependence of the magnetic three-gluon vertex zero crossing $\lambda_{A^{3}}^{\mathrm{M}}\left(p_{0}\right)=0$.

equations is the quadratic Casimir operator of the adjoint representation $C_{A}$. Furthermore, $C_{A}$ occurs only in combination with the coupling at the renormalization point $\alpha(\mu) \cdot C_{A} \equiv \tilde{\alpha}(\mu)$. Thus, it can be absorbed into a redefinition of the running coupling, or, equivalently, the scale of the theory. Therefore, the propagators are identical for all groups, and the different couplings can be obtained by a trivial rescaling with $C_{A}$. We emphasize that this is not a mere artifact of the approximation. Perturbatively, the beta function of the pure gauge theory has a trivial group dependence up to three loops; see, e.g., [127] for a recent discussion. See Sec. V for a discussion.

At low momenta, the electric and magnetic propagators show a qualitatively different behavior. While the magnetic gluon propagator decreases almost monotonously with increasing temperature, the electric propagator increases at small temperatures. At high temperatures, where the growth of thermal contributions to the mass becomes dominant, also the electric gluon propagator decreases; see also Sec. II C and in particular Fig. 3. For the magnetic gluon propagator we find agreement with the lattice results on the $10 \%$ accuracy level we expect from the truncation of the vertices. Furthermore, we see that the deviation takes its maximum for temperatures about the phase transition temperature, where we expect large-scale dynamical fluctuations to be most relevant. On the one hand, our truncation is tested maximally in this regime, and on the other hand discretization and finite volume effects in the lattice calculation are strongest there. In contradistinction to the very satisfactory situation for the magnetic propagator, we observe a significant deviation about the phase transition temperature $T_{c}$ for the electric gluon propagator. However, the agreement is very good for small and large temperatures. As discussed in great detail in Sec. II B and V, the deviation about $T_{c}$ can be explained by the missing nontrivial $\left\langle A_{0}\right\rangle$-background in the present calculation.

The ghost propagator agrees qualitatively, but deviates quantitatively, from the lattice results, as shown in Fig. 8(a). We discuss this point further in Sec. V. The ghost-gluon vertex is plotted in Fig. 8(b). Interestingly, it is weaker around the phase transition temperature than in the vacuum. At high temperatures it shows a broader and less pronounced bump than at zero temperature.

The gluonic vertex dressing functions are shown in Figs. 9 and 10 . The magnetic dressings of both vertices show scaling in the infrared. Contrarily, the corresponding electric components decouple at $p \approx 2 \pi T$ and become constant in the infrared. We show the position of the zero crossing of the magnetic three-gluon vertex dressing function as a function of temperature in Fig. 11. At small temperatures the zero crossing moves towards lower momenta as the temperature is increased, since the three-gluon vertex is stronger at small and intermediate momenta for small temperatures, cf., Fig. 9. At high temperatures, the magnetic zero crossing rises linearly with the temperature. In contrast, the zero crossing of the electric three-gluon vertex dressing function disappears at $T \approx 40 \mathrm{MeV}$. Similarly, the electric dressing of the four-gluon vertex undergoes a drastic change from zero to 
TABLE I. Scaling exponents. See Sec. II A for the definition of the symmetric momentum approximation and Appendix A for a comparison.

\begin{tabular}{lccc}
\hline \hline & & $\begin{array}{c}\text { Symmetric } \\
\text { momentum }\end{array}$ & Full momentum \\
& Simple vertices & approximation & dependence \\
\hline$d=4$ & $0.5953[32,33,36]$ & $0.567(3)$ & $0.576(5)[2]$ \\
$d=3$ & $0.3976[32]$ & $0.321(1)[100]$ & $\times$ \\
$d=4, T>0$ & $\times$ & $0.323(3)$ & $\times$ \\
\hline \hline
\end{tabular}

small temperatures, where scaling is lost, and goes on to increase with growing temperature.

At low momenta $p \ll 2 \pi T$ the dimension of the theory is effectively reduced and the magnetic dressings behave as they do in three dimensions. In the case of the scaling solution, all magnetic dressing functions scale with a power-law $[39,40,44]$,

$$
\lim _{p \rightarrow 0} \lambda^{(2 n, m)}(p) \propto\left(p^{2}\right)^{(n-m) \kappa+(1-n)\left(\frac{d}{2}-2\right)},
$$

where $2 n$ and $m$ is the number of ghost and gluon legs, respectively. Due to dimensional reduction, the temperature-independent scaling coefficient $\kappa$ is determined by three-dimensional Yang-Mills theory. Fitting the magnetic gluon propagators to (38) with $d=3$ at $p \ll 2 \pi T$, we find $\kappa=0.323(3)$. This agrees with the scaling exponent $\kappa=0.321(1)$ of the three-dimensional vacuum theory [100]. We summarize the different scaling coefficients in Table I.

\section{DISCUSSION}

In the previous section we have presented nonperturbative results obtained with the most comprehensive truncation within functional methods to date. The agreement of the magnetic propagator and the electric propagator for high temperatures is of the order of $10 \%$ in the momentum regime relevant for hadronic observables. These small deviations can be attributed to lattice artifacts, the relative scale setting uncertainty, and the systematic error within our truncation. The latter stems from incomplete momentum dependencies of the vertices and missing nonclassical tensors; see Appendixes A and D for estimates of their respective importance. The electric propagator deviates from the lattice results at temperatures about the phase transition temperature. The explanation has already been indicated in Sec. IV and is discussed below.

\section{A. Nontrivial backgrounds and their impact on electric and magnetic propagators}

A potential source of the discrepancy of the electric gluon propagator near the phase transition temperature is an insufficient order in our approximation scheme. However, such deviations of the electric gluon propagator from lattice results were already observed in much simpler truncations [62]. Furthermore, if truncation artifacts were the main source, we would expect larger discrepancies also in the magnetic gluon propagator.

In contrast to this, the electric propagator, which is closely related to the order parameter $L\left[\left\langle\bar{A}_{0}\right\rangle\right]$, is particularly sensitive to a nonvanishing background field [65]. As argued in Sec. II B, the nontrivial solution of the equation of motion, $\bar{A}_{0} \neq 0$, is important in the temperature regime (25), that is

$$
T \in\left(0.5 T_{c}, 1.3 T_{c}\right)
$$

This is exactly the temperature range where the deviations from the lattice results, which are evaluated on the equation of motion, are most pronounced. We expect a considerable improvement in the electric propagator if the correlation functions are evaluated on the nontrivial background. At this point, we want to emphasize that the observed deviations do not invalidate our results for the electric two-point correlator. It simply represents the correlation functions at a nonminimal configuration, cf. (18). Furthermore, these findings underline that Polyakov-enhanced low-energy effective models should be constructed in $\bar{A}_{0}$-backgrounds and the effective potential $V\left[\bar{A}_{0}\right]$ rather than Polyakov loop backgrounds and the Polyakov loop potential $V[L]$ : the electric propagators agree on the $10 \%$ level above $T \gtrsim 1.3 T_{c}$. This entails that the relevant background for the shifts in the Matsubara frequencies is $\left\langle\bar{A}_{0}\right\rangle$.

The above analysis is also important for the discussion of the comparison of the present results with $S U(2)$ and $S U$ (3) lattice simulations. As discussed in detail in the last Sec. IV, the gauge group enters only at very high orders of the approximation in an expansion of the effective action around vanishing background. Thus, our results depend only trivially on the gauge group. However, the gauge group, and in particular the universality class, enters via the Polyakov loop background, or, more precisely $\left\langle\bar{A}_{0}\right\rangle$. It has already been shown in $[74,80]$ that the different orders of the phase transition for $S U(2)$ and $S U(N>2)$ are encoded in the Polyakov loop potential $V\left[\bar{A}_{0}\right]$ and the respective expectation values $\nu$ in (24), rather than in the propagators. The Ising critical exponents for $S U(2)$ are also extracted from critical fluctuations encoded in the effective potential; see [75] for Yang-Mills theory in Polyakov gauge and [102] for Landau-gauge Yang-Mills theory. In [75,102] it also has been shown, that the critical fluctuations are the actual cause of the higher phase transition temperature in comparison to $S U(N>2)$. Thus, the gauge group dependence of the order of the phase transition and the value of transition temperature are to leading order caused by the effective potential, and hence by the related expansion about the physical ground state, i.e., $\left\langle\bar{A}_{0}\right\rangle$ in the current setting. 
We close the discussion of the propagators with the remark that the comparison of our results with the lattice results at small momenta $p^{2} \ll \Lambda_{\mathrm{QCD}}^{2}$ has to be taken with a grain of salt. The lattice results are of the decoupling type, while our results are of the scaling type. Consequently, possible nonperturbative gauge fixing effects have to be kept in mind; see, e.g., [128-131]. This concerns in particular the ghost propagator, shown in Fig. 8(a), which is more sensitive to the treatment of the Gribov copies than the gluon propagator [131].

\section{B. Debye mass and perturbative regime}

We find very good agreement of our nonperturbative Debye screening mass with two-loop hard thermal loop perturbation theory down to $T \approx 0.6 \mathrm{GeV}$; see Fig. 3. This remarkable agreement down to comparably low temperatures is in line with earlier findings; see, e.g., [86-94]. In general, perturbative resummation schemes have been found to be applicable at surprisingly large couplings. An explanation of this unexpectedly large range of validity can be given by the structural similarity of higher order perturbative resummation schemes and the nonperturbative resummations performed within functional methods. This opens the door for applications of functionally assisted analytic perturbative computations beyond the validity bounds of perturbation theory, in particular to the transport and kinetic realm of heavy ion collisions.

\section{Three-gluon vertex and its zero crossing}

The magnetic three-gluon vertex dressing function has been studied on the lattice [72] and with a semi-perturbative approximation of its DSE [73]. Both studies show a significant enhancement of the magnetic dressing at low momenta $p \approx 0.2 \mathrm{GeV}$ for temperatures just below the critical temperature. While we also observe this effect qualitatively, see Fig. 9, we find a much weaker enhancement. This is consistent with the finding that our electric gluon propagator is weaker than the electric lattice propagator, cf., Fig. 7. This electric propagator enters the triangle diagram in the three-gluon vertex equation, which yields a positive contribution to the dressing function [53]. Thus, a stronger electric propagator increases the strength of the magnetic three-gluon vertex.

At zero temperature, the three-gluon vertex shows a zero crossing in four as well as in three dimensions $[2,6,46,49,51-53,59,132-135]$. Analytical studies show that it is caused by the divergent ghost triangle diagram. We find that the zero crossing persists in the magnetic dressing function for all temperatures. This stands in line with [73] but in contrast to [72], where the lowest investigated momenta show a positive sign at temperatures somewhat below the critical temperature. Here, we present an analytical argument for the persistence of the magnetic zero crossing at all temperatures. The argument is first presented for a vanishing gluonic background and is based on the infrared dominance of ghost loops. Finally we discuss the case of nonvanishing gluonic backgrounds relevant for temperatures about $T_{c}$.

All gluonic diagrams are gapped below a certain scale, whereas the ghost triangle effectively behaves like the corresponding three-dimensional diagram for $p^{2} \ll(2 \pi T)^{2}$. Therefore, it causes a divergence in the magnetic threegluon vertex dressing function at low momenta for all temperatures, and thus, the magnetic zero crossing cannot vanish. At high temperatures, this zero crossing moves then to higher scales, which is in line with the high temperature limit and [72]. This qualitative argument is actually independent of the type of the solution, since the threedimensional ghost triangle diagram diverges with a powerlaw in the case of the scaling solution and linearly $[6,49,51]$ in the case of the decoupling solution. We find that the zero crossing of the electric component vanishes at a temperature of $T \approx 40 \mathrm{MeV}$. This can be understood by observing that the zero mode of the ghost triangle diagram, evaluated at zero external Matsubara frequencies, contributes to the magnetic three-gluon vertex dressing, but vanishes analytically if projected with the electric three-gluon vertex projection operator. Our numerical results show precisely the expected behavior; see Figs. 9 and 11.

We extend the argument to the case of nonvanishing backgrounds. They introduce a color structure in the ghost propagator and the ghost-gluon vertex. After diagonalization, we are left with gapped and ungapped modes in the ghost propagator, as well as background-dependent and background-independent (color) tensor structures in the ghost-gluon vertex. The remaining ungapped ghost modes couple to the latter tensor structure, which is nothing but the original tensor structure at vanishing background. Therefore, the background simply leads to a weakening of the infrared dominance by gapping some, but not all, ghost modes. Accordingly, the zero crossing moves towards smaller momenta, but does not disappear, in the presence of nontrivial backgrounds. Furthermore, for small temperatures $T / \Lambda_{\mathrm{QCD}} \rightarrow 0$, the gapping of the ghost occurs only at very small momenta $\vec{p}^{2} \lesssim(2 \pi T)^{2}$, and we are left with the temperature regime (25), in which a weakening of the infrared ghost dominance is to be expected. This structure is compatible with the results in [72], where no zero crossing was observed at temperatures about $T_{c}$ in the accessible momentum regime. In our opinion, it would therefore be interesting to extend the analysis of [72] to smaller momenta.

\section{SUMMARY AND OUTLOOK}

We have presented nonperturbative first-principles results for the finite-temperature Landau-gauge YangMills correlation functions, obtained from the functional renormalization group. Our comprehensive truncation of the effective action includes the computationally especially expensive magnetic and electric components of the purely 
gluonic vertices. We gauged our truncation by comparing to propagator results obtained in lattice simulations and found very good agreement for the magnetic gluon propagator. Our result for the Debye screening mass shows excellent agreement with two-loop hard thermal loop perturbation theory at high temperatures and the electric gluon propagator compares very well to lattice results for all temperatures except $T \in\left(0.5 T_{c}, 1.3 T_{c}\right)$. We have argued that the deviations in this regime are related to the different backgrounds used. Particular focus was also put on the fate of the zero crossing in the three-gluon vertex at finite temperature. In the electric component of the threegluon vertex we found the disappearance of the zero crossing at a very small temperature. The magnetic zero crossing also moves towards lower momenta for small temperatures but it never vanishes. At high temperatures, its position increases linearly with the temperature. We gave an analytic argument for the observed qualitative behavior of the zero crossing in the magnetic and electric components.

The presented first-principles results for the finitetemperature correlation functions of Yang-Mills theory form the foundation for a number of subsequent studies. First and foremost, the capability to perform nonperturbative first-principles studies of gauge theories at finite temperatures provides a crucial prerequisite for the investigation of the QCD phase structure. In particular, combining the advancements of this work with those of a recent calculation of the correlators of two-flavor QCD [3], will allow us to investigate the properties of quantum chromodynamics at finite temperature and density from first principles. Furthermore, the presented correlators can be used to compute thermodynamic quantities like the pressure, the shear viscosity, as well as the Polyakov loop potential and spectral functions, the latter being notoriously difficult to obtain. Additionally, it is suggestive to use the remarkable agreement of fully nonperturbative results with resummed perturbative results, in particular in the Debye mass, to devise functionally assisted analytic applications for the transport and kinetic regime in heavy ion collisions. Finally, we expect that improving the current investigation by including a nonvanishing background field and nonvanishing Matsubara modes will lead to the disappearance of the discrepancy in the electric gluon propagator near the phase transition temperature.

\section{ACKNOWLEDGMENTS}

We thank Lukas Corell, Ouraman Hajizadeh, Markus Q. Huber, Axel Maas, Richard Williams, and Nicolas Wink for discussions. This work is supported by ExtreMe Matter Institute (EMMI), Grant No. ERC-AdG-290623, the FWF through Erwin-Schrödinger-Stipendium No. J3507-N27, the Studienstiftung des deutschen Volkes, the DFG through Grant No. STR 1462/1-1, the BMBF Grant No. 05P12VHCTG, and is part of and supported by the DFG Collaborative Research Centre "SFB 1225 (ISOQUANT)." It is also supported in part by the Office of Nuclear Physics in the U.S. Department of Energy's Office of Science under Contract No. DEAC02-05CH11231.

\section{APPENDIX A: REGULATOR AND TRUNCATION DEPENDENCE}

The regulators in (31) are parametrized by

$$
\begin{aligned}
R_{k, \mu \nu}^{a b}(p) & =\tilde{Z}_{A, k}^{\mathrm{M}} r\left(p^{2} / k^{2}\right) p^{2} \delta^{a b} \Pi_{\mu \nu}^{\perp}(p), \\
R_{k}^{a b}(p) & =\tilde{Z}_{c, k} r\left(p^{2} / k^{2}\right) p^{2} \delta^{a b},
\end{aligned}
$$

where we dress the regulators with $\tilde{Z}_{A, k}^{\mathrm{M}}$ and $\tilde{Z}_{c, k}$ as in [2]. Since $\Pi_{\mu \nu}^{\perp}(p)=\Pi_{\mu \nu}^{\mathrm{M}}(p)+\Pi_{\mu \nu}^{\mathrm{E}}(p)$, (A1) implies the same regularization for electric and magnetic modes. Due to its advantages for the evaluation of thermodynamic quantities $[62,97]$ we use the exponential regulator shape function,

$$
r(x)=\frac{x^{m-1} e^{-x^{m}}}{1-e^{-x^{m}}},
$$

with $m=2$. This is in contrast to the vacuum calculations in [2], which were performed with a smoothed version of the flat regulator [104].

In Fig. 12, we show vacuum results obtained with the flat and the exponential regulator. Clearly, the results obtained with the symmetric-point approximation, defined by (13), agree very well. However, they show a higher bump than the lattice results. This is due to the symmetric-point approximation used for the vertices. This discrepancy vanishes if more momentum dependencies are included as shown in Fig. 12, cf. [2], for a thorough discussion. An extension of the current finite-temperature investigations

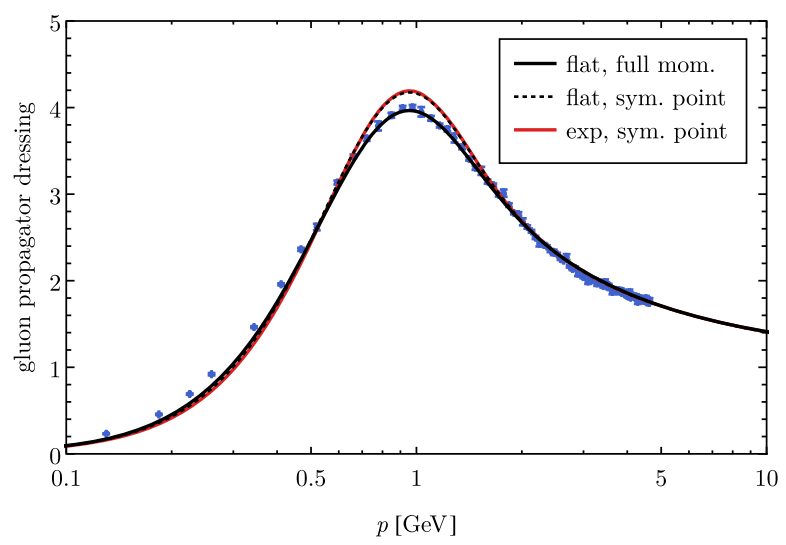

FIG. 12. Gluon propagator dressing obtained with the exponential regulator in comparison with dressings calculated with the flat regulator in [2] and $S U(3)$ lattice data [124]. The lattice results are renormalized as in [2]. Newer lattice results [125] agree with [124] if the largest physical volumes are compared. 
beyond the symmetric-point approximation is deferred to future work.

\section{APPENDIX B: TENSOR SPLITTING}

At vanishing Matsubara frequencies, not all tensors that are obtained by contracting the classical tensor structures with all possible combinations of electric and magnetic projectors are linearly independent; see Appendix C. Since we calculate the dressing functions at vanishing Matsubara mode, we can compute only a restricted set of dressings. This implies that we have to approximate the remaining degenerate dressing functions from this reduced set of dressings in order to obtain the correct UV behavior and to recover the vacuum results in the zero-temperature limit. We use

$$
\left[\Gamma_{A \bar{c} c}^{(3)}\right]_{\mu}^{a b c}(p, q)=\left[S_{A \bar{c} c}^{(3)}\right]_{\mu^{\prime}}^{a b c}(p, q)\left(\lambda_{\bar{c} c A}^{\mathrm{M}}(\bar{p}) \Pi_{\mu^{\prime} \mu}^{\mathrm{M}}+\lambda_{\bar{c} c A}^{\mathrm{M}}(\bar{p}) \Pi_{\mu^{\prime} \mu}^{\mathrm{E}}\right)
$$

for the ghost-gluon vertex,

$$
\begin{aligned}
{\left[\Gamma_{A^{3}}^{(3)}\right]_{\mu \nu \rho}^{a b c}(p, q)=} & {\left[S_{A^{3}}^{(3)}\right]_{\mu^{\prime} \nu^{\prime} \rho^{\prime}}^{a b c}(p, q) \cdot\left(\lambda_{A^{3}}^{\mathrm{M}}(\bar{p}) \Pi_{\mu^{\prime} \mu}^{\mathrm{M}} \Pi_{\nu^{\prime} \nu}^{\mathrm{M}} \Pi_{\rho^{\prime} \rho}^{\mathrm{M}}+\left(\lambda_{A^{3}}^{\mathrm{M}}(\bar{p}) \Pi_{\mu^{\prime} \mu}^{\mathrm{E}} \Pi_{\nu^{\prime} \nu}^{\mathrm{M}} \Pi_{\rho^{\prime} \rho}^{\mathrm{M}}+\text { perm. }\right)\right.} \\
& \left.+\left(\lambda_{A^{3}}^{\mathrm{E}}(\bar{p}) \Pi_{\mu^{\prime} \mu}^{\mathrm{E}} \Pi_{\nu^{\prime} \nu}^{\mathrm{E}} \Pi_{\rho^{\prime} \rho}^{\mathrm{M}}+\text { perm. }\right) \lambda_{A^{3}}^{\mathrm{M}}(\bar{p}) \Pi_{\mu^{\prime} \mu}^{\mathrm{E}} \Pi_{\nu^{\prime} \nu}^{\mathrm{E}} \Pi_{\rho^{\prime} \rho}^{\mathrm{E}}\right),
\end{aligned}
$$

for the three-gluon vertex and

$$
\begin{aligned}
{\left[\Gamma_{A^{4}}^{(4)}\right]_{\mu \nu \rho \sigma}^{a b c d}(p, q, r)=} & {\left[S_{A^{4}}^{(4)}\right]_{\mu^{\prime} \nu^{\prime} \rho^{\prime} \sigma^{\prime}}^{a c d} \cdot\left(\lambda_{A^{4}}^{\mathrm{M}}(\bar{p}) \Pi_{\mu^{\prime} \mu}^{\mathrm{M}} \Pi_{\nu^{\prime} \nu}^{\mathrm{M}} \Pi_{\rho^{\prime} \rho}^{\mathrm{M}} \Pi_{\sigma^{\prime} \sigma}^{\mathrm{M}}+\left(\lambda_{A^{4}}^{\mathrm{M}}(\bar{p}) \Pi_{\mu^{\prime} \mu}^{\mathrm{E}} \Pi_{\nu^{\prime} \nu}^{\mathrm{M}} \Pi_{\rho^{\prime} \rho}^{\mathrm{M}} \Pi_{\sigma^{\prime} \sigma}^{\mathrm{M}}+\text { perm. }\right)\right.} \\
& +\left(\lambda_{A^{4}}^{\mathrm{E}}(\bar{p}) \Pi_{\mu^{\prime} \mu}^{\mathrm{E}} \Pi_{\nu^{\prime} \nu}^{\mathrm{E}} \Pi_{\rho^{\prime} \rho}^{\mathrm{M}} \Pi_{\sigma^{\prime} \sigma}^{\mathrm{M}}+\text { perm. }\right)+\left(\lambda_{A^{4}}^{\mathrm{M}}(\bar{p}) \Pi_{\mu^{\prime} \mu}^{\mathrm{E}} \Pi_{\nu^{\prime} \nu}^{\mathrm{E}} \Pi_{\rho^{\prime} \rho}^{\mathrm{E}} \Pi_{\sigma^{\prime} \sigma}^{\mathrm{M}}+\text { perm. }\right) \\
& +\left(\lambda_{A^{4}}^{\mathrm{M}}(\bar{p}) \Pi_{\mu^{\prime} \mu}^{\mathrm{E}} \Pi_{\nu^{\prime} \nu}^{\mathrm{E}} \Pi_{\rho^{\prime} \rho}^{\mathrm{E}} \Pi_{\sigma^{\prime} \sigma}^{\mathrm{E}}\right),
\end{aligned}
$$

for the four-gluon vertex. Here and in the following we leave the momentum arguments implicit. Although the dressing functions of some tensors coincide in our approximation, we explicitly show the splitting to make the construction of the approximation apparent. The magnetic dressing functions appear in more than one tensor structure, and we evaluate them by projecting onto the purely magnetic tensors structure for every vertex; see Appendix D. Due to the $O(4)$-symmetry of the vacuum, this approximation becomes exact for large momenta $p^{2} \gg(2 \pi T)^{2}$, which are not affected by finite-temperature effects. In the limit of vanishing Matsubara frequencies, the dimension of the tensor space is reduced; see Appendix C. Therefore, this approximation is very good also for small momenta $p^{2} \lesssim(2 \pi T)^{2}$. Hence, the approximations used in (B1)-(B3) affect only intermediate Matsubara modes, which are only slightly influenced by finite temperature effects; see Sec. II A.

\section{APPENDIX C: TENSOR DEGENERACY}

We generalize the classical tensor structures to finite temperature by attaching all combinations of magnetic and electric projection operators; see (B1)-(B3). However, contracting the electric ghost-gluon vertex with itself and evaluating it at vanishing Matsubara modes yields

$$
\left.\left[S_{A \bar{c} c}^{(3)}\right]_{\mu}^{a b c} \Pi_{\mu \mu^{\prime}}^{\mathrm{E}}\left[S_{A \bar{c} c}^{(3)}\right]_{\mu^{\prime}}^{a b c}\right|_{\left\{n_{i}=0\right\}}=0 .
$$

Hence, the electric component of the ghost-gluon vertex disappears in the limit of vanishing Matsubara frequencies. Similarly, we find for the three-gluon vertex,

$$
\begin{aligned}
& {\left.\left[S_{A^{3}}^{(3)}\right]_{\mu \nu \rho}^{a b c} \Pi_{\mu \mu^{\prime}}^{\mathrm{E}} \Pi_{\nu \nu^{\prime}}^{\mathrm{M}} \Pi_{\rho \rho^{\prime}}^{\mathrm{M}}\left[S_{A^{3}}^{(3)}\right]_{\mu^{\prime} \nu^{\prime} \rho^{\prime}}^{a b c}\right|_{\left\{n_{i}=0\right\}}=0,} \\
& {\left.\left[S_{A^{3}}^{(3)}\right]_{\mu \nu \rho}^{a b c} \Pi_{\mu \mu^{\prime}}^{\mathrm{E}} \Pi_{\nu \nu^{\prime}}^{\mathrm{E}} \Pi_{\rho \rho^{\prime}}^{\mathrm{E}}\left[S_{A^{3}}^{(3)}\right]_{\mu^{\prime} \nu^{\prime} \rho^{\prime}}^{a b c}\right|_{\left\{n_{i}=0\right\}}=0,}
\end{aligned}
$$

and for the four-gluon vertex,

$$
\begin{aligned}
& {\left.\left[S_{A^{4}}^{(4)}\right]_{\mu \nu \rho \sigma}^{a b c d} \Pi_{\mu \mu^{\prime}}^{\mathrm{E}} \Pi_{\nu \nu^{\prime}}^{\mathrm{M}} \Pi_{\rho \rho^{\prime}}^{\mathrm{M}} \Pi_{\sigma \sigma^{\prime}}^{\mathrm{M}}\left[S_{A^{4}}^{(4)}\right]_{\mu^{\prime} \nu^{\prime} \rho^{\prime} \sigma^{\prime}}^{a b d}\right|_{\left\{n_{i}=0\right\}}=0,} \\
& {\left.\left[S_{A^{4}}^{(4)}\right]_{\mu \nu \rho \sigma}^{a b c d} \Pi_{\mu \mu^{\prime}}^{\mathrm{E}} \Pi_{\nu \nu^{\prime}}^{\mathrm{E}} \Pi_{\rho \rho^{\prime}}^{\mathrm{E}} \Pi_{\sigma \sigma^{\prime}}^{\mathrm{M}}\left[S_{A^{4}}^{(4)}\right]_{\mu^{\prime} \nu^{\prime} \rho^{\prime} \sigma^{\prime}}^{a b c d}\right|_{\left\{n_{i}=0\right\}}=0,} \\
& {\left.\left[S_{A^{4}}^{(4)}\right]_{\mu \nu \rho \sigma}^{a b c d} \Pi_{\mu \mu^{\prime}}^{\mathrm{E}} \Pi_{\nu \nu^{\prime}}^{\mathrm{E}} \Pi_{\rho \rho^{\prime}}^{\mathrm{E}} \Pi_{\sigma \sigma^{\prime}}^{\mathrm{E}}\left[S_{A^{4}}^{(4)}\right]_{\mu^{\prime} \nu^{\prime} \rho^{\prime} \sigma^{\prime}}^{a c c d}\right|_{\left\{n_{i}=0\right\}}=0 .}
\end{aligned}
$$

Thus, for $p^{2} \ll(2 \pi T)^{2}$ the classical vertex dressings are fully described by the remaining basis tensors, to wit, those with only magnetic legs and those with exactly two electric legs.

\section{APPENDIX D: PROJECTING THE FLOW EQUATIONS}

The tensor bases for the propagators as well as for the ghost-gluon vertex are complete, and therefore the projection onto the dressings is unique. For the gluonic vertices we do not take the full transverse tensor bases into account. 
Consequently, already in the vacuum, any projection is an approximation that relies on the assumption that nonincluded basis elements are small. If the flows are projected onto their electric and magnetic components, the incompleteness of the bases can lead to intricate complications. The reason is that the magnetic and electric projection operators can yield differing contributions from nonclassical tensor structures that are created by quantum fluctuations. As an immediate consequence, the magnetic and electric dressings differ then by momentum dependent terms. This effect occurs already at vanishing temperature, and is therefore in contradiction with the $O(4)$-symmetry of the vacuum. If one uses a complete basis, projecting with magnetic and electric projection operators does not spoil the $O(4)$-symmetry although the projection operators themselves are not $O(4)$-symmetric.

In the following two subsections we discuss in detail the quantitative relevance of these effects caused by the incomplete bases for the gluonic vertices. In order to disentangle genuine finite-temperature contributions from these projection artifacts, we consider only vacuum flows. By splitting the projection into electric and magnetic components and comparing them to the $O(4)$-symmetric projection, we are able to quantify these basis artifacts. Unfortunately, we find that the emergence of certain nonclassical tensors yields sizeable artifacts on the dressing of the classical tensor structure of the four-gluon vertex. As discussed in detail in this and the following two Appendixes $\mathrm{E}$ and $\mathrm{F}$, implementing a proper treatment of these artifacts of the incomplete bases turns out to be vital to obtain the correct UV behavior and cutoff independence of the finite-temperature results.

\section{Three-gluon vertex}

We project onto the magnetic and electric components of the three-gluon vertex by

$$
\begin{aligned}
& \lambda_{A^{3}}^{\mathrm{M}}= \frac{\left[S_{A^{3}}^{(3)}\right]_{\mu \nu \rho}^{a b c} \Pi_{\mu \mu^{\prime}}^{\mathrm{M}} \Pi_{\nu \nu^{\prime}}^{\mathrm{M}} \Pi_{\rho \rho^{\prime}}^{\mathrm{M}}\left[\Gamma_{A^{3}}^{(3)}\right]_{\mu^{\prime} \nu^{\prime} \rho^{\prime}}^{a b c}}{\left[S_{A^{3}}^{(3)}\right]_{\mu \nu \rho}^{a b c} \Pi_{\mu \mu^{\prime}}^{\mathrm{M}} \Pi_{\nu \nu^{\prime}}^{\mathrm{M}} \Pi_{\rho \rho^{\prime}}^{\mathrm{M}}\left[S_{A^{3}}^{(3)}\right]_{\mu^{\prime} \nu^{\prime} \rho^{\prime}}^{a b c}} \\
& \lambda_{A^{3}}^{\mathrm{E}}=\frac{\left[S_{A^{3}}^{(3)}\right]_{\mu \nu \rho}^{a b c} \Pi_{\mu \mu^{\prime}}^{\mathrm{E}} \Pi_{\nu \nu^{\prime}}^{\mathrm{E}} \Pi_{\rho \rho^{\prime}}^{\mathrm{M}}\left[\Gamma_{A^{3}}^{(3)}\right]_{\mu^{\prime} \nu^{\prime} \rho^{\prime}}^{a b c}}{\left[S_{A^{3}}^{(3)}\right]_{\mu \nu \rho}^{a b c} \Pi_{\mu \mu^{\prime}}^{\mathrm{E}} \Pi_{\nu \nu^{\prime}}^{\mathrm{E}} \Pi_{\rho \rho^{\prime}}^{\mathrm{M}}\left[S_{A^{3}}^{(3)}\right]_{\mu^{\prime} \nu^{\prime} \rho^{\prime}}^{a b c}}
\end{aligned}
$$

as generalization of the vacuum projection

$$
\lambda_{A^{3}}=\frac{\left[S_{A^{3}}^{(3)}\right]_{\mu \nu \rho}^{a b c} \Pi_{\mu \mu^{\prime}}^{\perp} \Pi_{\nu \nu^{\prime}}^{\perp} \Pi_{\rho \rho^{\prime}}^{\perp}\left[\Gamma_{A^{3}}^{(3)}\right]_{\mu^{\prime} \nu^{\prime} \rho^{\prime}}^{a c}}{\left[S_{A^{3}}^{(3)}\right]_{\mu \nu \rho}^{a b c} \Pi_{\mu \mu^{\prime}}^{\perp} \Pi_{\nu \nu^{\prime}}^{\perp} \Pi_{\rho \rho^{\prime}}^{\perp}\left[S_{A^{3}}^{(3)}\right]_{\mu^{\prime} \nu^{\prime} \rho^{\prime}}^{a b c}}
$$

In explicit numerical checks we find that the projections (D1) and (D2) agree to the per mille level at $T=0$ and therefore also for $k \gg 2 \pi T$. We conclude that our projection is not sensitive to the possible emergence of nonclassical tensors structures in the three-gluon vertex. This is also consistent with the sub-leading importance of nonclassical tensor structures found in earlier three-gluon vertex studies [53].

\section{Four-gluon vertex}

We project onto the vacuum dressing function with

$$
\lambda_{A^{4}}=\frac{\left[S_{A^{4}}^{(4)}\right]_{\mu \nu \rho \sigma}^{a b c d} \Pi_{\mu \mu^{\prime}}^{\perp} \Pi_{\nu \nu^{\prime}}^{\perp} \Pi_{\rho \rho^{\prime}}^{\perp} \Pi_{\sigma \sigma^{\prime}}^{\perp}\left[\Gamma_{A^{4}}^{(4)}\right]_{\mu^{\prime} \nu^{\prime} \rho^{\prime} \sigma^{\prime}}^{a b c d}}{\left[S_{A^{4}}^{(4)}\right]_{\mu \nu \rho \sigma}^{a b c d} \Pi_{\mu \mu^{\prime}}^{\perp} \Pi_{\nu \nu^{\prime}}^{\perp} \Pi_{\rho \rho^{\prime}}^{\perp} \Pi_{\sigma \sigma^{\prime}}^{\perp}\left[S_{A^{4}}^{(4)}\right]_{\mu^{\prime} \nu^{\prime} \rho^{\prime} \sigma^{\prime}}^{a b c d}} .
$$

Assuming vanishing nonclassical tensor structures, this generalizes to

$$
\begin{aligned}
& \lambda_{A^{4}}^{\mathrm{M}}=\frac{\left[S_{A^{4}}^{(4)}\right]_{\mu \nu \rho \sigma}^{a b c d} \Pi_{\mu \mu^{\prime}}^{\mathrm{M}} \Pi_{\nu \nu^{\prime}}^{\mathrm{M}} \Pi_{\rho \rho^{\prime}}^{\mathrm{M}} \Pi_{\sigma \sigma^{\prime}}^{\mathrm{M}}\left[\Gamma_{A^{4}}^{(4)}\right]_{\mu^{\prime} \nu^{\prime} \rho^{\prime} \sigma^{\prime}}^{a b c d}}{\left[S_{A^{4}}^{(4)}\right]_{\mu \nu \rho \sigma}^{a b c d} \Pi_{\mu \mu^{\prime}}^{\mathrm{M}} \Pi_{\nu \nu^{\prime}}^{\mathrm{M}} \Pi_{\rho \rho^{\prime}}^{\mathrm{M}} \Pi_{\sigma \sigma^{\prime}}^{\mathrm{M}}\left[S_{A^{4}}^{(4)}\right]_{\mu^{\prime} \nu^{\prime} \rho^{\prime} \sigma^{\prime}}^{a b d}} \\
& \lambda_{A^{4}}^{\mathrm{E}}=\frac{\left[S_{A^{4}}^{(4)}\right]_{\mu \nu \rho \sigma}^{a b c d} \Pi_{\mu \mu^{\prime}}^{\mathrm{E}} \Pi_{\nu \nu^{\prime}}^{\mathrm{E}} \Pi_{\rho \rho^{\prime}}^{\mathrm{M}} \Pi_{\sigma \sigma^{\prime}}^{\mathrm{M}}\left[\Gamma_{A^{4}}^{(4)}\right]_{\mu^{\prime} \nu^{\prime} \rho^{\prime} \sigma^{\prime}}^{a b d},}{\left[S_{A^{4}}^{(4)}\right]_{\mu \nu \rho \sigma}^{a b c d} \Pi_{\mu \mu^{\prime}}^{\mathrm{E}} \Pi_{\nu \nu^{\prime}}^{\mathrm{E}} \Pi_{\rho \rho^{\prime}}^{\mathrm{M}} \Pi_{\sigma \sigma^{\prime}}^{\mathrm{M}}\left[S_{A^{4}}^{(4)}\right]_{\mu^{\prime} \nu^{\prime} \rho^{\prime} \sigma^{\prime}}^{a}},
\end{aligned}
$$

for the magnetic and the electric components. If the only tensor generated by the flow were the classical one,

$$
\left[\Gamma_{A^{4}}^{(4)}\right]_{\mu \nu \rho \sigma}^{a b c d} \propto\left[S_{A^{4}}^{(4)}\right]_{\mu \nu \rho \sigma}^{a b c d}=f^{a b n} f^{c d n} \delta_{\mu \rho} \delta_{\nu \sigma}+\text { perm. }
$$

the projections (D3) and (D4) would yield $\lambda_{A^{4}}=\lambda_{A^{4}}^{\mathrm{M}}=\lambda_{A^{4}}^{\mathrm{E}}$. However, this equality can be spoiled by the presence of nonclassical tensors, which are in general created by the flow equation. Consider, for example, the following $O(4)$ - and Bose-symmetric nonclassical tensor:

$$
\begin{aligned}
& {\left[\Gamma_{A^{4}, \mathrm{ncl}}^{(4)}\right]_{\mu \nu \rho \sigma}^{a b c d}(p, q, r, s)} \\
& \quad=\left(f^{a b n} f^{c d n} \cdot(q+s)_{\mu}(q+s)_{\rho}(p+r)_{\nu}(p+r)_{\sigma}\right)+\text { perm. }
\end{aligned}
$$

Inserting (D6) into (D3) and (D4) yields differing contributions to the dressing functions $\lambda_{A^{4}}, \lambda_{A^{4}}^{\mathrm{M}}$, and $\lambda_{A^{4}}^{\mathrm{E}}$. Therefore, $O(4)$-invariance is lost due to the incompleteness of the basis that was used to construct the projection operators, (D3) and (D4).

In Fig. 13, we show the vacuum flows of the four-gluon vertex obtained with different projection operators and identical vacuum vertices on the right hand side of the flow equation. In contrast to the three-gluon vertex, we find a considerable difference in the resulting momentum dependence of the projections (D3) and (D4). We conclude, that sizeable nonclassical tensors, which affect the difference between the magnetic and electric projection operators, are generated. As an immediate consequence, the $O(4)$ symmetric limit at $T \rightarrow 0$ is spoiled by the presence of these tensors since $\lambda_{A^{4}}^{\mathrm{M}}(T=0) \neq \lambda_{A^{4}}^{\mathrm{E}}(T=0)$. 


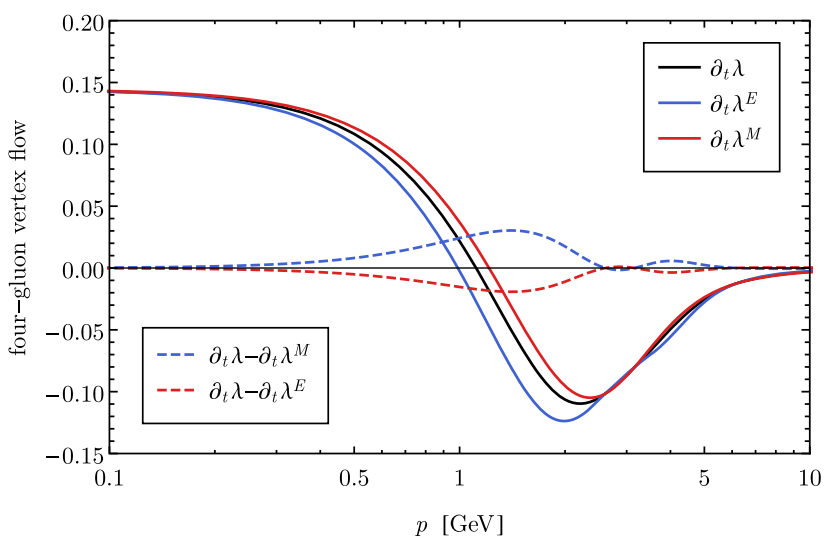

FIG. 13. Four-gluon vertex vacuum flows from different projection operators, (D3) and (D4), and their differences at the RG scale $k=2 \mathrm{GeV}$.

A simple estimate of the unphysical projection artifacts of these nonclassical tensors is given by the vacuum differences of the projections (D3) and (D4),

$$
\begin{aligned}
& \partial_{t}\left[\lambda_{A^{4}}-\lambda_{A^{4}}^{\mathrm{M}}(T=0)\right], \\
& \partial_{t}\left[\lambda_{A^{4}}-\lambda_{A^{4}}^{\mathrm{E}}(T=0)\right],
\end{aligned}
$$

which are also shown in Fig. 13. Assuming that this unphysical difference depends only mildly on the temperature, a natural strategy to account for this artifact is to subtract (D7) from the finite-temperature flows. However, there is an additional complication in the case of the scaling solution. The vertex dressings obey a power law behavior at small momenta, see (38), and the corresponding exponent changes as one goes from the vacuum to finite temperature. Although this behavior of the correlators at very small momenta does not affect any observables, it has to be taken into account when subtracting (D7) from the finite-temperature flows. Consequently, we modify the flows of the magnetic and electric components by

$\partial_{t} \lambda_{A^{4}}^{\mathrm{M}}(T)=\partial_{t} \lambda_{A^{4}}^{\mathrm{M}}(T)+\theta_{\epsilon}\left(k, k_{c}\right) \partial_{t}\left[\lambda_{A^{4}}-\lambda_{A^{4}}^{\mathrm{M}}(T=0)\right]$,

$\partial_{t} \lambda_{A^{4}}^{\mathrm{E}}(T)=\partial_{t} \lambda_{A^{4}}^{\mathrm{E}}(T)+\theta_{\epsilon}\left(k, k_{c}\right) \partial_{t}\left[\lambda_{A^{4}}-\lambda_{A^{4}}^{\mathrm{E}}(T=0)\right]$.

The purpose of the smoothed step function,

$$
\theta_{\epsilon}\left(k, k_{c}\right)=\frac{1}{1+\exp \left[\frac{1}{\epsilon}\left(1-\frac{k}{k_{c}}\right)\right]},
$$

is to provide a transition from the corrected flows to the pure finite-temperature flows with the correct scaling behavior at very low momenta. We set the transition scale $k_{c}$ to

$$
k_{c}=\min \left(\lambda_{c} 2 \pi T, \Lambda_{c}\right)
$$

which is defined in terms of the parameters $\Lambda_{c}$ and $\lambda_{c}$. The modified dressings fulfill

$$
\lim _{T \rightarrow 0} \lambda_{A^{4}}^{\mathrm{M}}(T)=\lim _{T \rightarrow 0} \lambda_{A^{4}}^{\mathrm{E}}(T)=\lambda_{A^{4}}
$$

This guarantees that we recover the vacuum results in the limit of vanishing temperature while our best estimates for the basis artifacts are subtracted above the transition scale $k_{c}$.

In order to investigate the influence of the precise value of the transition scale, we vary $\Lambda_{c}$ and $\lambda_{c}$ in reasonable ranges. Since temperature effects are expected to be small at momentum scales $k \geq 2 \pi T, \lambda_{c}$ should be of order unity and we vary it from 1 to 2 . Furthermore, the gapping scale of the gluon propagator gives us an estimate on the scale below which no phenomenologically important effects are to be expected. Consequently, we vary $\Lambda_{c}$ between the

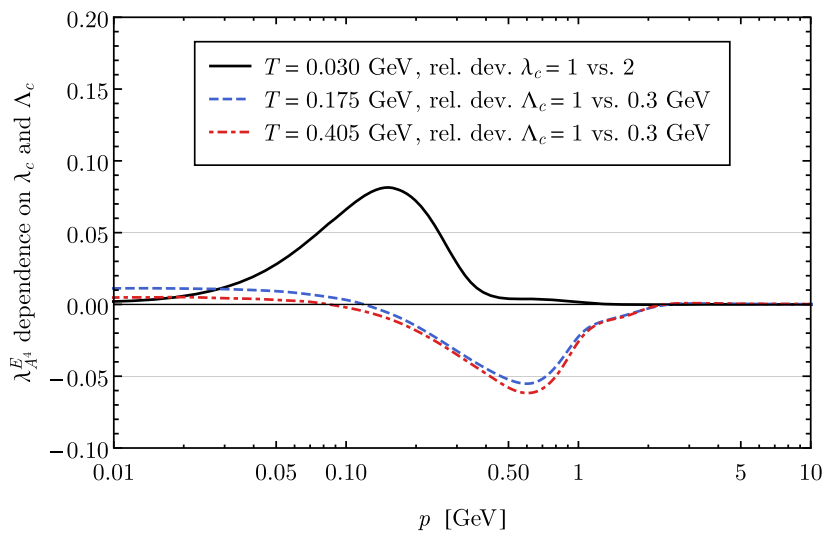

(b) Electric dressing.

FIG. 14. Relative deviations, e.g., $\left(\lambda_{A^{4}}^{\mathrm{M}}\left(\lambda_{c}=1\right)-\lambda_{A^{4}}^{\mathrm{M}}\left(\lambda_{c}=2\right)\right) / \lambda_{A^{4}}^{\mathrm{M}}\left(\lambda_{c}=1\right)$, of the four-gluon vertex dressings, (B3), calculated with different parameters in the smoothed theta function (D9). Depending on the temperature, the dressings depend either on $\lambda_{c}$ or $\Lambda_{c}$; see (D10). 
location of the maxima of the gluon propagator and the gluon propagator dressing, i.e., $\Lambda_{c} \in[0.3,1] \mathrm{GeV}$. We find only a mild (10\%) dependence of the four-gluon vertex dressings on these parameters as shown in Fig. 14. Since the four-gluon vertex is the least important of all classical tensors in the self-consistently coupled system, we find that the dependence of all other dressings on these parameters is even smaller. For example, the induced uncertainty on the electric gluon propagator is at most $3 \%$, but for a wide range of temperatures and momenta it is even smaller than $0.5 \%$. In all cases the dependence on the smoothing parameter, $\epsilon=0.05$, is negligible.

\section{APPENDIX E: VACUUM LIMIT}

We constructed the truncation such that it converges to the symmetric-point approximation used in [2] in the vacuum limit, $T \rightarrow 0$. In Fig. 15(a), we show the gluon propagator for a range of small temperatures. We clearly see that the magnetic as well as the electric propagators approach the vacuum propagator in the zero temperature limit. In particular, for each temperature there exists a threshold momentum above which the magnetic, the electric and the vacuum dressings agree. This is not only a strong check of our code but also shows the validity of (B1)-(B3) at $k \ll T$ as well as the consistency of our $O(4)$-symmetric momentum approximations, i.e., (13). Similarly, the magnetic and electric dressing functions of the other vertices become degenerate in the vacuum limit. As discussed in the next appendix, this behavior allows us to significantly reduce the computational effort.

\section{APPENDIX F: INITIAL SCALE}

The regulator suppresses quantum as well as thermal fluctuations below the regulator scale $k$. Therefore, the

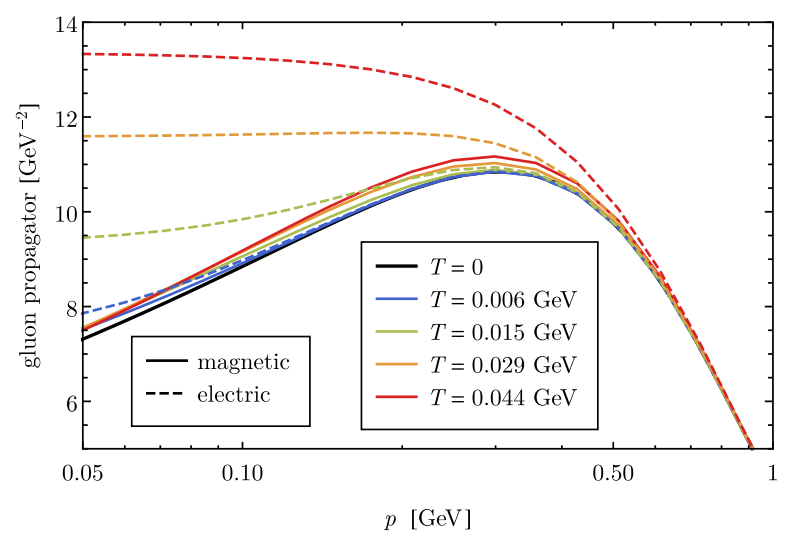

(a) Convergence towards the vacuum results. The plot shows the degeneracy of the magnetic and the electric gluon propagators for $p \gtrsim 2 \pi T$ temperature-dependent generalized effective action $\Gamma_{k}(T)$ agrees with the zero-temperature effective action as long as temperature fluctuations are suppressed,

$$
\Gamma_{k}(T)=\Gamma_{k}(T=0) \quad \text { if } 2 \pi T \ll k .
$$

This property enables us to reduce the computational effort by one to two orders of magnitude. First we compute the $T=0$ effective action, starting at a large perturbative scale of typically $k=\Lambda=60 \mathrm{GeV}$ from the classical action. To obtain the temperature-dependent 1PI effective action, we integrate the flow equation starting from the effective average action $\Gamma_{\Lambda_{T}}(T)=\Gamma_{\Lambda_{T}}(T=0)$ at a lower, temperature-dependent cutoff scale,

$$
\Lambda_{T}=\max \left(\lambda 2 \pi T, \Lambda_{T}^{\min }\right) .
$$

Here, $\Lambda_{T}^{\min }$ has been introduced to avoid the interference of the lowered starting scale with the dynamical mass generation of the gluon. This is necessary, because the scaling condition forces us to modify the input at $\Lambda_{T}$ by a gluon mass term; see Appendix G. Consequently, we choose $\Lambda_{T}^{\min }$ as the scale where the vacuum gluon propagator dressing becomes maximal, i.e., $\Lambda_{T}^{\min }=0.955 \mathrm{GeV}$. We show the dependence of the longitudinal gluon propagator on the physical start scale $\lambda$ in Fig. 15(b). The gluon propagator as well as all other quantities do not depend on the start scale for $\lambda \geq 2$. In our numerical computation we use $\lambda=4$ although $\lambda=2$ is sufficient, as argued in Appendix G.

To demonstrate the advantage of the temperaturedependent initial scale, we consider the numerical vacuum integration,

$$
\int_{q}=\int \frac{d^{4} q}{(2 \pi)^{4}}=\int_{0}^{L} \frac{d q}{(2 \pi)^{4}} q^{3} \int d \Omega .
$$

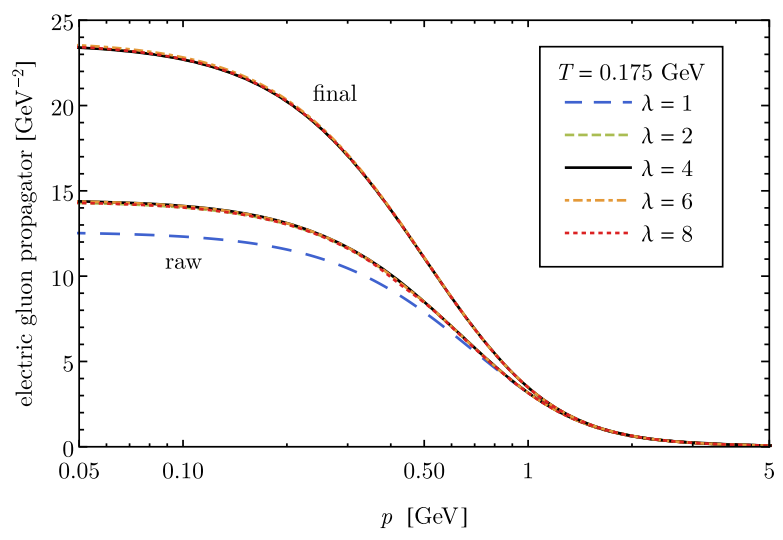

(b) Gluon propagator obtained with different initial scales $\Lambda_{T}=\lambda 2 \pi T$. See App. G for the definition of raw and final. We do not show the final propagator for $\lambda=1$ since in this case the start scale $\Lambda_{T}=2 \pi T$ lies below the correction scale $k_{T}=4 \pi T$, cf. (G2).

FIG. 15. Vacuum limit and initial scale independence of gluon propagator, (9). 
Numerically, it is advantageous to choose a $k$-dependent numerical cutoff $L=l k$, where $l=3$ is sufficient for the exponential regulator due to the regulator derivative appearing in all diagrams. This persists in the Matsubara formalism and we can limit the summation to frequencies $\omega=2 \pi T n$ smaller than $L=l k$,

$$
y_{q}=\int_{0}^{L} \frac{d q}{(2 \pi)^{3}} q^{2} \int d \Omega T \sum_{n}^{w \leq L} .
$$

Thus, the number of required integrand evaluations grows linearly with $k$ as well as with $1 / T$. For small temperatures, the increased number of evaluations due to the growing number of small Matsubara modes is therefore compensated by the shrinking initial scale, at least down to $T=\Lambda_{T}^{\min } /(2 \pi \lambda)$.

\section{APPENDIX G: GLUON MASS PARAMETER}

The gluon mass parameter, $m_{\Lambda}^{2} \propto \alpha(\Lambda) \Lambda^{2}$, is fixed at the cutoff scale $k=\Lambda$, which is far bigger than the temperature scale, $\Lambda \gg 2 \pi T$. As discussed in Sec. III B, this parameter is determined by the modified Slavnov-Taylor identity that is difficult to solve numerically with the required quadratic precision. In addition, a truncation to the combined system of mSTI and flow has to be self-consistent to quadratic precision. At vanishing temperature, we have therefore determined the gluon mass term by requiring a solution of the scaling type.

Temperature effects are suppressed exponentially for the used regulators; see [105] and Sec. III A. Hence, the initial conditions for the flow at $k=\Lambda$ converge exponentially to that at vanishing temperature. However, in the present scaling solution the initial conditions compensate for the violation of the modified BRST-symmetry during the flow, and in particular at low cutoff scales. Therefore, we expect a temperature-dependent change of the initial conditions for compensating temperature-dependent truncation artifacts at low scales. Keeping this in mind, we extend the BRSTconsistent fine-tuning of the initial conditions to finite temperature,

$\Gamma_{A A, k=\Lambda_{T}}^{(2), \mathrm{M} \text {,raw }}(p)=\Gamma_{A A, k=\Lambda_{T}}^{(2) \text { E,raw }}(p)=\Gamma_{A A, k=\Lambda_{T}}^{(2), T=0}(p)+\Delta m_{T}^{2}$.

The temperature-dependent part of the gluon mass parameter $\Delta m_{T}^{2}$ is fixed such that we obtain infrared scaling in the purely magnetic sector; see Sec. III B. Its sole purpose is to adjust the modified BRST symmetry as in the $T=0$ case. Requiring scaling fixes $\Delta m_{T}^{2}$ uniquely. While adjusting the correct infrared behavior, this introduces truncation artifacts in the UV. The RG-relevant part of the temperaturedependence at large momentum has to vanish identically. It is uniquely removed with
$\Gamma_{A A, k=0}^{(2), \mathrm{M} / \mathrm{E}}=\Gamma_{A A, k=0}^{(2), \mathrm{M} / \mathrm{E} \text {,raw }}-\left(\Gamma_{A A, k=k_{T}}^{(2), \mathrm{M} / \mathrm{E} \text {,raw }}-\Gamma_{A A, k=k_{T}}^{(2), T=0}\right)$.

Here, $k_{T} \approx 4 \pi T \leq \Lambda_{T}$ is the scale above which temperature effects are virtually absent, i.e.,

$$
\Gamma_{A A, k \geq k_{T}}^{(2), \mathrm{M} \text {,raw }}=\Gamma_{A A, k \geq k_{T}}^{(2), \mathrm{E} \text {,raw }} .
$$

Note that (G2) keeps the physical temperature-dependent polynomially suppressed large momentum corrections; see [62]. Equation (G2) removes in particular $\Delta m_{T}^{2}$ from the final result. Moreover, the mass correction (G1) leads to modifications of the flows due to the back coupling of the changed gluon mass parameter. Consequently, the subtraction (G2) removes back-coupling artifacts that are accumulated during the integration of the flow from $\Lambda_{T}$ to $k_{T}$. In the case $\Lambda_{T}=k_{T}$, no back-coupling artifacts are created at scales larger than $k_{T}$ and the correction becomes trivial,

$$
\Gamma_{A A, k=k_{T}}^{(2), \mathrm{M} / \mathrm{E}, \mathrm{raw}}-\Gamma_{A A, k=k_{T}}^{(2), T=0}=\Delta m_{T}^{2}
$$

We demonstrate in Fig. 15(b) that neither the raw nor the final gluon propagators, obtained with (G2), depend on the initial cutoff scale $\Lambda_{T}$. Thus, $\Lambda_{T}=k_{T}$ is the numerically least demanding and most stable choice that includes all thermal fluctuations. Note that (G1) modifies the magnetic and electric propagators identically. Thus, the electric mass is an observable at vanishing cutoff.

In order to assess the effect of the temperature-dependent tuning of the gluon mass parameter (G1), we compare the raw with the final propagators in Fig. 16. We observe a sizeable influence of the correction on the final result. We plot the raw and the final magnetic gluon propagator each normalized by the magnetic lattice propagator in Fig. 17. Since the lattice data have to be renormalized for each temperature separately, agreement is always found at the corresponding momentum scale; see Appendix H. At lower scales, the raw propagator quickly deviates from the lattice results. Contrarily, the final propagator shows better agreement, which we interpret as support for our subtraction procedure $(\mathrm{G} 2)$.

\section{APPENDIX H: SCALE SETTING AND RENORMALIZATION}

We set the scale by rescaling our internal units such that the bump position of the gluon propagator dressing lies at $p_{\max } \equiv 0.955 \mathrm{GeV}$, and thus coincides with the bump position of the vacuum lattice results from [124]; see Fig. 12.

The temperatures of the $S U(2)$ lattice results from $[69,123]$ are given in terms of the critical temperature. In order to compare, we use $T_{c}^{S U(2)}=0.7091 \sqrt{\sigma}=312 \mathrm{MeV}$ [136] to convert the temperature into units of $\mathrm{GeV}$, where the 


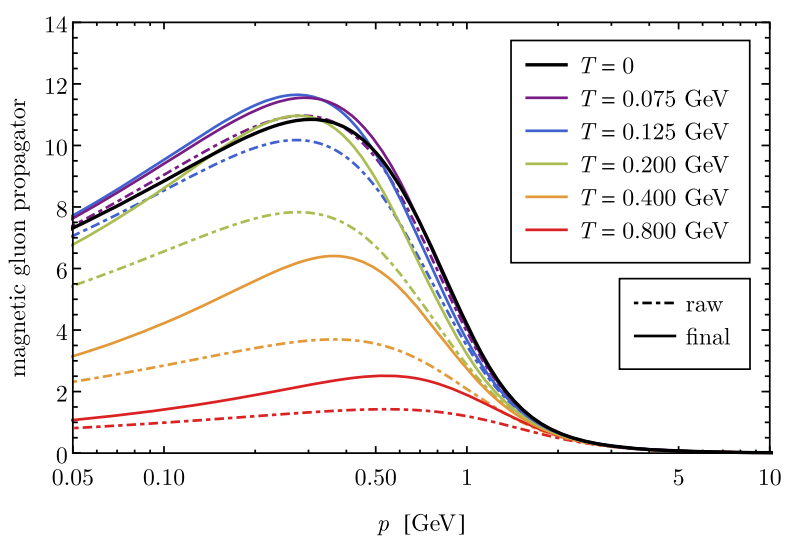

(a) Magnetic gluon propagator $1 /\left(p^{2} Z_{A}^{\mathrm{M}}(p)\right)$.

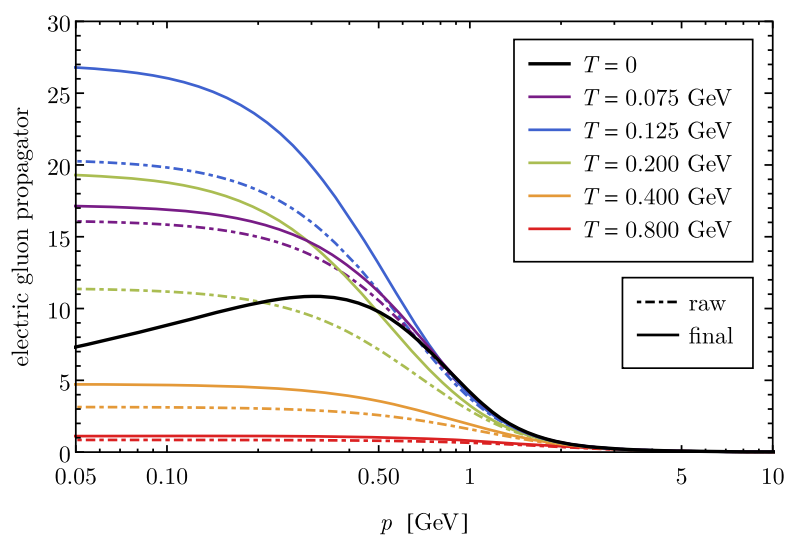

(b) Electric gluon propagator $1 /\left(p^{2} Z_{A}^{\mathrm{E}}(p)\right)$.

FIG. 16. Electric and magnetic gluon propagators with and without mass subtraction procedure, (G2).

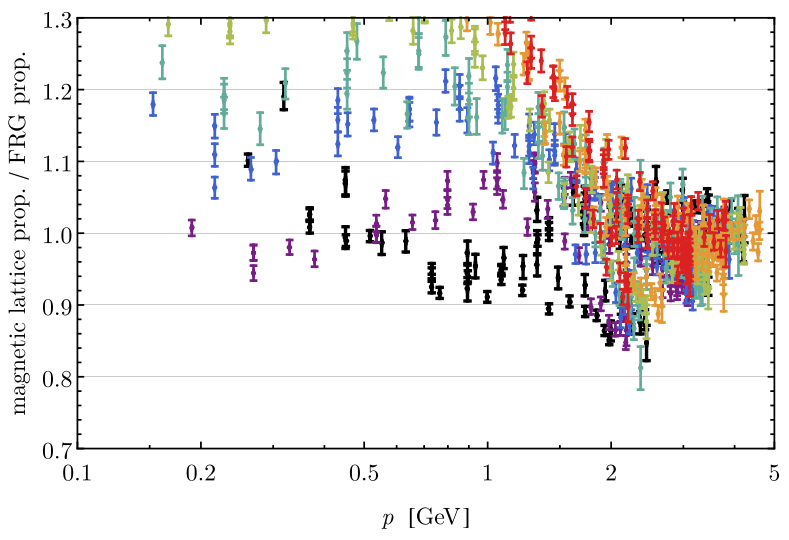

(a) Raw propagator.

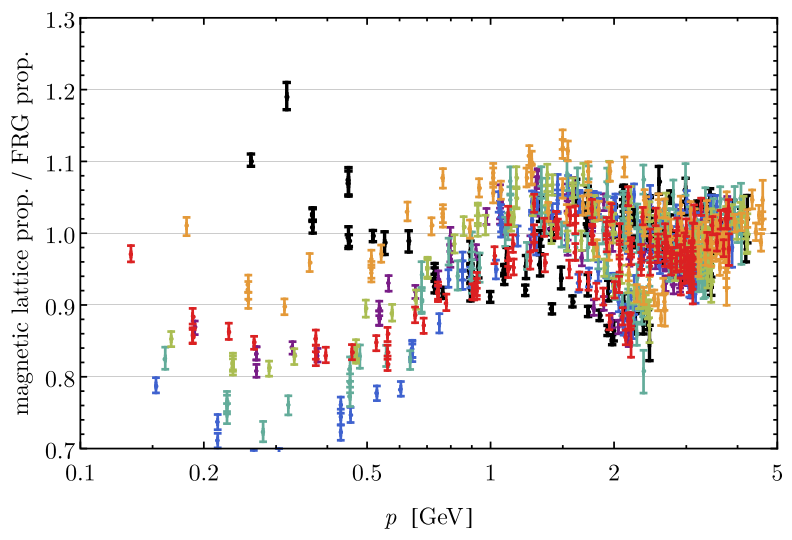

(b) Final propagator.

FIG. 17. Magnetic $S U(2)$ lattice propagator [69,123] over FRG propagator; see Fig. 6(a) for the color coding.

string tension $\sigma$ is given by $\sigma=0.440 \mathrm{GeV}^{2}$. These lattice results need to be renormalized for each temperature separately. We determine the temperature-dependent renormalization constants by fitting all lattice points above $p \geq \max (2 \pi T, 1 \mathrm{GeV})$ to our results.

The $S U(3)$ lattice results from [71] do not include the vacuum case $T=0$. Therefore, we allow for a scale mismatch by introducing a temperature-independent relative scale factor $r_{s}$, in addition to the temperatureindependent wave function renormalization constant $z_{L}$. We determine $r_{s}$ and $z_{L}$ by fitting the magnetic gluon dressing function, $1 /\left(z_{L} Z_{A}^{\mathrm{M}}\left(r_{s} T, r_{s} p\right)\right)$, simultaneously for all temperatures to all lattice points above $p \geq 0.5 \mathrm{GeV}$. Subsequently, we use $r_{s}$ and $z_{L}$ to rescale the magnetic as well as the electric lattice propagators to our data. We find the relative scale mismatch $r_{s}-1$ to be small, of the order of $2 \%$. The temperatures in [71] are given in units of GeV. In order to simplify the discussion, we convert the temperatures into units of the critical temperature, using their value for the $S U$ (3) phase transition temperature, $T_{c}=270 \mathrm{MeV}$.

\section{APPENDIX I: SCREENING MASS}

In this appendix we describe the extraction of the screening masses shown in Sec. IIC and provide the propagators in position space; see Fig. 18. In general, the thermal propagators at large distances show a combination of an exponential and an algebraic decay,

$$
\lim _{x \rightarrow \infty} G_{T}^{\mathrm{E}}(x)=c_{a} x^{1-4 \kappa}+c_{e} \exp \left(-m_{s} x\right)
$$

where $G_{T}^{\mathrm{E}}(x)$ is the Fourier transformed zero mode of the electric gluon propagator (26); see Sec. IIC. The algebraic decay originates from the infrared scaling at vanishing temperature with $1-4 \kappa$ being the scaling exponent in position space, cf., also (38). At low enough temperatures $T \ll T_{c}$, we see remnants of this zero-temperature algebraic part. At higher temperatures we have $c_{a}=0$. 


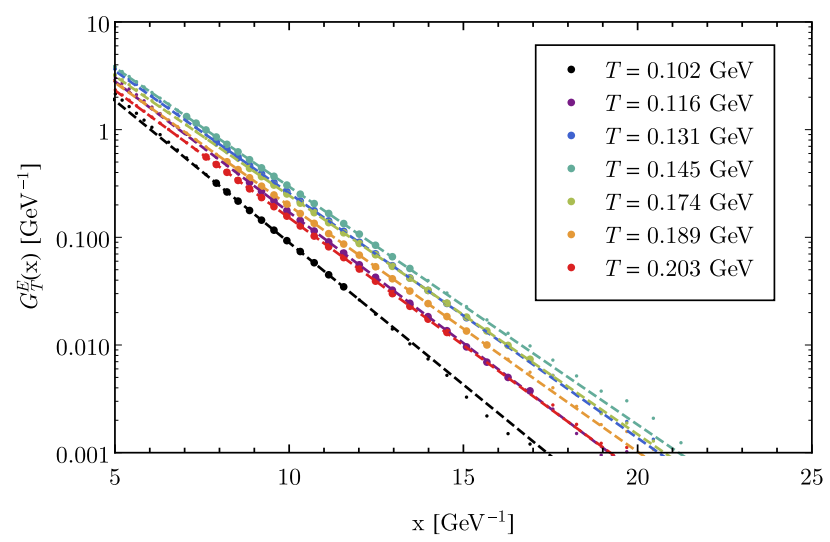

(a) $G_{T}^{\mathrm{E}}(x)$ for low temperatures.

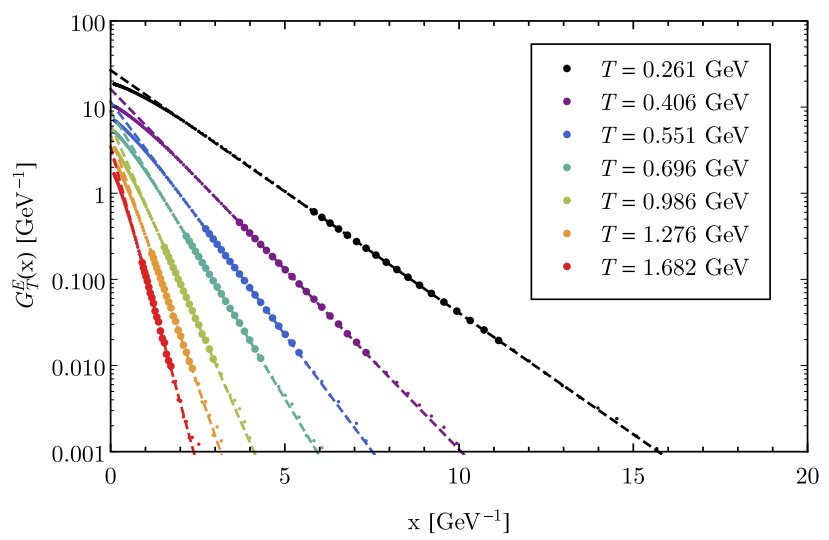

(b) $G_{T}^{\mathrm{E}}(x)$ for high temperatures.

FIG. 18. Exponential tail of the Fourier transformed electric propagator, $G_{T}^{\mathrm{E}}(x)$; see (26). The dashed lines are fits of (I1) with $c_{a}=0$, i.e., (27), to the large points. Small points show $G_{T}^{\mathrm{E}}(x)$ beyond the fit regions. The fitted screening masses as a function of temperature are shown in Fig. 3.

In Fig. 18, we show $G_{T}^{\mathrm{E}}(x)$ for low (left panel) and high (right panel) temperatures. For all shown temperatures, the exponential decay is apparent as linear regime. For even lower temperatures, the linear regime shrinks considerably due to the algebraic decay, and we need a higher numerical precision for extracting the sub-leading exponential decay. This is clearly seen in the lowest temperature shown, $T=0.102 \mathrm{GeV}$.
[1] M. Mitter, J. M. Pawlowski, and N. Strodthoff, Phys. Rev. D 91, 054035 (2015).

[2] A. K. Cyrol, L. Fister, M. Mitter, J. M. Pawlowski, and N. Strodthoff, Phys. Rev. D 94, 054005 (2016).

[3] A. K. Cyrol, M. Mitter, J. M. Pawlowski, and N. Strodthoff, arXiv:1706.06326.

[4] D. Binosi, L. Chang, J. Papavassiliou, and C. D. Roberts, Phys. Lett. B 742, 183 (2015).

[5] R. Williams, C. S. Fischer, and W. Heupel, Phys. Rev. D 93, 034026 (2016).

[6] M. Q. Huber, Phys. Rev. D 93, 085033 (2016).

[7] J. Berges, N. Tetradis, and C. Wetterich, Phys. Rep. 363, 223 (2002).

[8] J. M. Pawlowski, Ann. Phys. (Amsterdam) 322, 2831 (2007).

[9] H. Gies, Lect. Notes Phys. 852, 287 (2012).

[10] B.-J. Schaefer and J. Wambach, Phys. Part. Nucl. 39, 1025 (2008).

[11] O. J. Rosten, Phys. Rep. 511, 177 (2012).

[12] J. Braun, J. Phys. G 39, 033001 (2012).

[13] L. von Smekal, Nucl. Phys. B, Proc. Suppl. 228, 179 (2012).

[14] R. Alkofer and L. von Smekal, Phys. Rep. 353, 281 (2001).

[15] C. D. Roberts and S. M. Schmidt, Prog. Part. Nucl. Phys. 45, S1 (2000).

[16] C. S. Fischer, J. Phys. G 32, R253 (2006).

[17] C. S. Fischer and R. Alkofer, Phys. Rev. D 67, 094020 (2003).
[18] C. S. Fischer, A. Maas, and J. M. Pawlowski, Ann. Phys. (Amsterdam) 324, 2408 (2009).

[19] D. Binosi and J. Papavassiliou, Phys. Rep. 479, 1 (2009).

[20] A. Maas, Phys. Rep. 524, 203 (2013).

[21] Ph. Boucaud, J. P. Leroy, A. Le Yaouanc, J. Micheli, O. Pène, and J. Rodríguez-Quintero, Few Body Syst. 53, 387 (2012).

[22] G. Eichmann, H. Sanchis-Alepuz, R. Williams, R. Alkofer, and C. S. Fischer, Prog. Part. Nucl. Phys. 91, 1 (2016).

[23] J. Braun, L. Corell, A. K. Cyrol, W.-J. Fu, M. Leonhardt, M. Mitter, J. M. Pawlowski, M. Pospiech, F. Rennecke, N. Strodthoff, and N. Wink (fQCD Collaboration) (unpublished).

[24] L. M. Haas, R. Stiele, J. Braun, J. M. Pawlowski, and J. Schaffner-Bielich, Phys. Rev. D 87, 076004 (2013).

[25] T. K. Herbst, M. Mitter, J. M. Pawlowski, B.-J. Schaefer, and R. Stiele, Phys. Lett. B 731, 248 (2014).

[26] J. Braun, L. Fister, J. M. Pawlowski, and F. Rennecke, Phys. Rev. D 94, 034016 (2016).

[27] F. Rennecke, Phys. Rev. D 92, 076012 (2015).

[28] W.-J. Fu and J. M. Pawlowski, Phys. Rev. D 92, 116006 (2015).

[29] M. Heller and M. Mitter, Phys. Rev. D 94, 074002 (2016).

[30] W.-j. Fu, J. M. Pawlowski, F. Rennecke, and B.-J. Schaefer, Phys. Rev. D 94, 116020 (2016).

[31] L. von Smekal, R. Alkofer, and A. Hauck, Phys. Rev. Lett. 79, 3591 (1997). 
[32] D. Zwanziger, Phys. Rev. D 65, 094039 (2002).

[33] C. Lerche and L. von Smekal, Phys. Rev. D 65, 125006 (2002).

[34] C. S. Fischer, R. Alkofer, and H. Reinhardt, Phys. Rev. D 65, 094008 (2002).

[35] H. Gies, Phys. Rev. D 66, 025006 (2002).

[36] J. M. Pawlowski, D. F. Litim, S. Nedelko, and L. von Smekal, Phys. Rev. Lett. 93, 152002 (2004).

[37] C. S. Fischer and H. Gies, J. High Energy Phys. 10 (2004) 048.

[38] R. Alkofer, C. S. Fischer, and F. J. Llanes-Estrada, Phys. Lett. B 611, 279 (2005).

[39] C.S. Fischer and J. M. Pawlowski, Phys. Rev. D 75, 025012 (2007).

[40] M. Q. Huber, R. Alkofer, C. S. Fischer, and K. Schwenzer, Phys. Lett. B 659, 434 (2008).

[41] R. Alkofer, M. Q. Huber, and K. Schwenzer, Phys. Rev. D 81, 105010 (2010).

[42] A. C. Aguilar, D. Binosi, and J. Papavassiliou, Phys. Rev. D 78, 025010 (2008).

[43] Ph. Boucaud, J. P. Leroy, A. Le Yaouanc, J. Micheli, O. Pène, and J. Rodríguez-Quintero, J. High Energy Phys. 06 (2008) 099.

[44] C. S. Fischer and J. M. Pawlowski, Phys. Rev. D 80, 025023 (2009).

[45] M. Tissier and N. Wschebor, Phys. Rev. D 82, 101701 (2010).

[46] M. Tissier and N. Wschebor, Phys. Rev. D 84, 045018 (2011).

[47] M. Q. Huber and L. von Smekal, J. High Energy Phys. 04 (2013) 149

[48] A. C. Aguilar, D. Ibáñez, and J. Papavassiliou, Phys. Rev. D 87, 114020 (2013).

[49] M. Peláez, M. Tissier, and N. Wschebor, Phys. Rev. D 88, 125003 (2013).

[50] M. Quandt, H. Reinhardt, and J. Heffner, Phys. Rev. D 89, 065037 (2014).

[51] A. Aguilar, D. Binosi, D. Ibáñez, and J. Papavassiliou, Phys. Rev. D 89, 085008 (2014).

[52] A. Blum, M. Q. Huber, M. Mitter, and L. von Smekal, Phys. Rev. D 89, 061703 (2014).

[53] G. Eichmann, R. Williams, R. Alkofer, and M. Vujinovic, Phys. Rev. D 89, 105014 (2014).

[54] J. Gracey, Phys. Rev. D 90, 025014 (2014).

[55] J. A. Gracey, Phys. Rev. D 90, 025011 (2014).

[56] D. Binosi, D. Ibáñez, and J. Papavassiliou, J. High Energy Phys. 09 (2014) 059.

[57] A. K. Cyrol, M. Q. Huber, and L. von Smekal, Eur. Phys. J. C 75, 102 (2015).

[58] M. Q. Huber, D. R. Campagnari, and H. Reinhardt, Phys. Rev. D 91, 025014 (2015).

[59] A. Athenodorou, D. Binosi, P. Boucaud, F. De Soto, J. Papavassiliou, J. Rodriguez-Quintero, and S. Zafeiropoulos, Phys. Lett. B 761, 444 (2016).

[60] U. Reinosa, J. Serreau, M. Tissier, and N. Wschebor, Phys. Rev. D 96, 014005 (2017).

[61] A. Maas, J. Wambach, B. Gruter, and R. Alkofer, Eur. Phys. J. C 37, 335 (2004).

[62] L. Fister and J. M. Pawlowski, arXiv:1112.5440.
[63] M. Quandt and H. Reinhardt, Phys. Rev. D 92, 025051 (2015).

[64] U. Reinosa, J. Serreau, M. Tissier, and N. Wschebor, Phys. Rev. D 89, 105016 (2014).

[65] U. Reinosa, J. Serreau, M. Tissier, and A. Tresmontant, Phys. Rev. D 95, 045014 (2017).

[66] A. Cucchieri, A. Maas, and T. Mendes, Phys. Rev. D 75, 076003 (2007).

[67] C. S. Fischer, A. Maas, and J. A. Muller, Eur. Phys. J. C 68, 165 (2010).

[68] R. Aouane, V. G. Bornyakov, E. M. Ilgenfritz, V. K. Mitrjushkin, M. Muller-Preussker, and A. Sternbeck, Phys. Rev. D 85, 034501 (2012).

[69] A. Maas, J. M. Pawlowski, L. von Smekal, and D. Spielmann, Phys. Rev. D 85, 034037 (2012).

[70] A. Cucchieri, D. Dudal, T. Mendes, and N. Vandersickel, arXiv:1202.0639.

[71] P. J. Silva, O. Oliveira, P. Bicudo, and N. Cardoso, Phys. Rev. D 89, 074503 (2014).

[72] L. Fister and A. Maas, Phys. Rev. D 90, 056008 (2014).

[73] M. Q. Huber, EPJ Web Conf. 137, 07009 (2017).

[74] J. Braun, H. Gies, and J. M. Pawlowski, Phys. Lett. B 684, 262 (2010).

[75] F. Marhauser and J. M. Pawlowski, arXiv:0812.1144.

[76] J. Braun, L. M. Haas, F. Marhauser, and J. M. Pawlowski, Phys. Rev. Lett. 106, 022002 (2011).

[77] K. Fukushima and K. Kashiwa, Phys. Lett. B 723, 360 (2013).

[78] L. Fister and J. M. Pawlowski, Phys. Rev. D 88, 045010 (2013).

[79] T. K. Herbst, J. Luecker, and J. M. Pawlowski, arXiv: 1510.03830 .

[80] J. Braun, A. Eichhorn, H. Gies, and J. M. Pawlowski, Eur. Phys. J. C 70, 689 (2010).

[81] A. Dumitru, Y. Guo, Y. Hidaka, C. P. K. Altes, and R. D. Pisarski, Phys. Rev. D 86, 105017 (2012).

[82] U. Reinosa, J. Serreau, M. Tissier, and N. Wschebor, Phys. Rev. D 91, 045035 (2015).

[83] U. Reinosa, J. Serreau, M. Tissier, and N. Wschebor, Phys. Rev. D 93, 105002 (2016).

[84] M. Quandt and H. Reinhardt, Phys. Rev. D 94, 065015 (2016).

[85] K. Fukushima and V. Skokov, Prog. Part. Nucl. Phys. 96, 154 (2017).

[86] U. M. Heller, F. Karsch, and J. Rank, Phys. Rev. D 57, 1438 (1998).

[87] S. Datta and S. Gupta, Phys. Lett. B 471, 382 (2000).

[88] A. Cucchieri, F. Karsch, and P. Petreczky, Phys. Rev. D 64, 036001 (2001).

[89] A. Nakamura, T. Saito, and S. Sakai, Phys. Rev. D 69, 014506 (2004).

[90] J. O. Andersen, M. Strickland, and N. Su, Phys. Rev. Lett. 104, 122003 (2010).

[91] J. O. Andersen, M. Strickland, and N. Su, J. High Energy Phys. 08 (2010) 113.

[92] P. B. Arnold and L. G. Yaffe, Phys. Rev. D 52, 7208 (1995).

[93] E. Braaten and A. Nieto, Phys. Rev. Lett. 76, 1417 (1996).

[94] E. Braaten and A. Nieto, Phys. Rev. D 53, 3421 (1996). 
[95] M. Haas, L. Fister, and J. M. Pawlowski, Phys. Rev. D 90, 091501 (2014).

[96] N. Christiansen, M. Haas, J. M. Pawlowski, and N. Strodthoff, Phys. Rev. Lett. 115, 112002 (2015).

[97] L. Fister, Ph.D. thesis, Heidelberg University, 2012.

[98] E.-M. Ilgenfritz, J. M. Pawlowski, A. Rothkopf, and A. Trunin, Eur. Phys. J. C 78, 127 (2018).

[99] P. J. Silva, O. Oliveira, D. Dudal, and M. Roelfs, Acta Phys. Pol. B Proc. Suppl. 10, 995 (2017).

[100] L. Corell, A. K. Cyrol, M. Mitter, J. M. Pawlowski, and N. Strodthoff (to be published).

[101] L. Corell, Master's thesis, University of Heidelberg, 2016.

[102] F. Spallek, Master's thesis, University of Heidelberg, 2011.

[103] C. Wetterich, Phys. Lett. B 301, 90 (1993).

[104] D. F. Litim, Phys. Lett. B 486, 92 (2000).

[105] L. Fister and J. M. Pawlowski, Phys. Rev. D 92, 076009 (2015).

[106] U. Ellwanger, Phys. Lett. B 335, 364 (1994).

[107] U. Ellwanger, M. Hirsch, and A. Weber, Z. Phys. C 69, 687 (1996).

[108] M. D'Attanasio and T. R. Morris, Phys. Lett. B 378, 213 (1996).

[109] D. F. Litim and J. M. Pawlowski, Phys. Lett. B 435, 181 (1998).

[110] Y. Igarashi, K. Itoh, and H. So, Prog. Theor. Phys. 106, 149 (2001).

[111] Y. Igarashi, K. Itoh, and J. M. Pawlowski, J. Phys. A 49, 405401 (2016).

[112] M. Q. Huber and J. Braun, Comput. Phys. Commun. 183, 1290 (2012).

[113] A. K. Cyrol, M. Mitter, and N. Strodthoff, Comput. Phys. Commun. C 219, 346 (2017).

[114] J. Vermaseren, arXiv:math-ph/0010025.

[115] J. Kuipers, T. Ueda, J. A. M. Vermaseren, and J. Vollinga, Comput. Phys. Commun. 184, 1453 (2013).

[116] J. Kuipers, T. Ueda, and J. A. M. Vermaseren, Comput. Phys. Commun. 189, 1 (2015).
[117] B. Ruijl, T. Ueda, and J. Vermaseren, arXiv:1707.06453.

[118] K. Ahnert and M. Mulansky, http://www.boost.org/doc/ libs/1_60_0/libs/numeric/odeint/doc/html/index.html.

[119] S. G. Johnson, Cubature (multi-dimensional integration), http://ab-initio.mit.edu/wiki/index.php/Cubature.

[120] J. Berntsen, T. O. Espelid, and A. Genz, ACM Trans. Math. Softw. 17, 437 (1991).

[121] A. C. Genz and A. Malik, J. Comput. Appl. Math. 6, 295 (1980).

[122] M. Tentyukov and J. A. M. Vermaseren, Comput. Phys. Commun. 181, 1419 (2010).

[123] A. Maas (private communication).

[124] A. Sternbeck, E. M. Ilgenfritz, M. Muller-Preussker, A. Schiller, and I. L. Bogolubsky, Proc. Sci., LAT2006 (2006) 076 [arXiv:hep-lat/0610053].

[125] A. G. Duarte, O. Oliveira, and P. J. Silva, Phys. Rev. D 94, 014502 (2016).

[126] T. van Ritbergen, A. N. Schellekens, and J. A. M. Vermaseren, Int. J. Mod. Phys. A 14, 41 (1999).

[127] F. Herzog, B. Ruijl, T. Ueda, J. A. M. Vermaseren, and A. Vogt, J. High Energy Phys. 02 (2017) 090.

[128] A. Maas, Phys. Lett. B 689, 107 (2010).

[129] A. Sternbeck and M. Müller-Preussker, Phys. Lett. B 726, 396 (2013).

[130] A. Maas, Phys. Rev. D 93, 054504 (2016).

[131] A. Maas, Ann. Phys. (Amsterdam) 387, 29 (2017).

[132] A. Cucchieri, A. Maas, and T. Mendes, Phys. Rev. D 74, 014503 (2006).

[133] A. Cucchieri, A. Maas, and T. Mendes, Phys. Rev. D 77, 094510 (2008).

[134] A. G. Duarte, O. Oliveira, and P. J. Silva, Phys. Rev. D 94, 074502 (2016).

[135] P. Boucaud, F. De Soto, J. Rodriguez-Quintero, and S. Zafeiropoulos, Phys. Rev. D 95, 114503 (2017).

[136] B. Lucini, M. Teper, and U. Wenger, J. High Energy Phys. 01 (2004) 061. 